\title{
Clinical pharmacy practice in the care of Chronic Kidney Disease patients: a systematic review
}

\author{
Fatma Al Raiisi ${ }^{1} \cdot$ Derek Stewart $^{1} \cdot$ Fernando Fernandez-Llimos $^{2} \cdot$ Teresa M. Salgado $^{3} \cdot$ Moustafa Fahmy Mohamed $^{4}$. \\ Scott Cunningham ${ }^{1} \mathbb{D}$
}

Received: 11 October 2018 / Accepted: 27 March 2019 / Published online: 9 April 2019

(c) The Author(s) 2019

\begin{abstract}
Background Clinical pharmacy services have potential to contribute significantly to the multidisciplinary team providing safe, effective and economic care for patients. Given recent practice developments (e.g. polypharmacy reviews and pharmacist prescribing) there is a need to provide a current synthesis of the evidence base for characteristics and outcomes of clinical pharmacy practice in chronic kidney disease patients. Aim of the review To critically appraise, synthesise and present the available evidence of the characteristics (structures and processes) and outcomes of clinical pharmacy practice as part of the multidisciplinary care of patients with chronic kidney disease. Method PubMed, International Pharmaceutical Abstracts (IPA), Cumulative Index to Nursing and Allied Health Literature (CINAHL), Medline and Scopus were searched for peer reviewed papers using improved search strategy. Included studies were quality assessed using Downs and Black tool for controlled studies and the mixed methods appraisal tool for all controlled and non-controlled studies. Data were extracted and synthesised using a narrative approach. Screening, quality assessment and data extraction were performed by two independent researchers. Ethics approval was not required. Results Forty-seven studies were identified from a variety of countries, with 31 based in a hospital setting. Controlled study designs were employed in 20, with only ten of these using randomisation. Resources available for service provision were poorly reported in all papers. Positive impact on clinical outcomes included significant improvement in parathyroid hormone, blood pressure, haemoglobin and creatinine clearance. Pharmacists identified 5302 drug related problems in 2933 patients and made 3160 recommendations with acceptance rates up to 95\%. Impact on humanistic outcomes was shown through improvement in health related quality of life and patient satisfaction. Economic benefits arose from significant cost savings through pharmaceutical care provision. Conclusion While there is some evidence of positive impact on clinical, humanistic and economic outcomes, this evidence is generally of low quality and insufficient volume. While the existing evidence is in favour of pharmacists' involvement in the multidisciplinary team providing care to patients with chronic kidney disease, more high-quality research is warranted.
\end{abstract}

Keywords Chronic kidney disease $\cdot$ Clinical pharmacy $\cdot$ Pharmacist $\cdot$ Systematic review

Electronic supplementary material The online version of this article (https://doi.org/10.1007/s11096-019-00816-4) contains supplementary material, which is available to authorized users.

Scott Cunningham

s.cunningham@rgu.ac.uk

1 School of Pharmacy \& Life Sciences, Robert Gordon University, Aberdeen, Scotland, UK

2 Research Institute for Medicines and Pharmaceutical Sciences (iMed.UL), Faculty of Pharmacy, University of Lisbon, Lisbon, Portugal
3 Department of Pharmacotherapy \& Outcomes Science, Center for Pharmacy Practice Innovation, Virginia Commonwealth University School of Pharmacy, Richmond, VA, USA

4 Oman Pharmacy Institute, Ministry of Health, Muscat, Oman 


\section{Impacts on practice}

- Understanding fully the structures, processes and relevant outcomes associated with clinical roles of pharmacists is essential to make best use of resource for optimal patient care.

- There has been a significant volume of research of the clinical role of pharmacists in Chronic Kidney Disease, but it is of limited detail and quality.

- There is a need for agreed standard sets of outcomes for clinical pharmacy practice and research in chronic kidney disease.

\section{Introduction}

Chronic Kidney Disease (CKD) continues to be a global concern with a high risk of mortality, frequent hospitalisation and reduced life expectancy [1]. Most patients have co-morbid conditions such as cardiovascular and mineral bone diseases [2]. Clinical pharmacy services have the potential to contribute significantly to the multidisciplinary team providing safe, effective and economic care [3]. Key clinical pharmacy roles in the multidisciplinary care of CKD patients were described by two renal pharmacy consultants Mason and Bakus in 2010 [4]. These roles included specific areas such as managing anaemia, renal mineral bone disease and hypertension, as well as more general medicines selection and review [4]. Another major role pharmacists can play is to contribute to renal drug cost management [5]. An emerging role is the potential for the pharmacist to prescribe and modify medicines, which has now been implemented into practice in the United Kingdom (UK), United States (USA) and New Zealand [6]. There is a need to establish the evidence base of the impact of clinical pharmacy in the care of CKD patients. In 2012, Salgado et al. published a systematic review which included synthesis of the peer reviewed literature up to March 2010 [7]. The original review identified 37 studies (38 articles), involving 4743 participants. Majority of the papers were of uncontrolled design (80\%) [7]. Twentyone articles $(55.3 \%)$ reported outcome measures and process indicators, 4 (10.5\%) reported only outcome measures, thirteen $(34.2 \%)$ reported only process indicators and none reported structures [7]. Pharmacists identified 2683 drug-related problems in 1209 patients. The results from controlled studies (average quality score $0.57, \mathrm{SD}=0.10$ ) reported that pharmacists' interventions reduced all-cause hospitalisations, reduced the incidence of end-stage renal disease or death in patients with diabetic nephropathy, improved management of anaemia, blood pressure, calcium and phosphate parameters and lipid management [7]. The uncontrolled studies included in the original review shown positive impact of pharmacists' interventions on the reduction of transplant rejections and fewer adverse events [7]. The reviews main limitations were selection and language bias which might affect the quality of the systematic review. Salgado et al. concluded that the evidence of pharmacists' interventions in patients with CKD is scarce, of variable quality and with heterogeneous outcomes [7]. Since the publication of the original review by Salgado et al., the prescribing practice has continually developed with new services and models of care being developed and embedded into clinical pharmacy practice. Hence, there is a need to update and extend the review. Given developments in clinical pharmacy globally, it is likely that further research has been reported thus an upto-date synthesis is warranted.

\section{Aim of the review}

The aim of this review was to critically appraise, synthesise and present the available evidence for the structures, processes and related outcomes of clinical pharmacy practice as part of the multidisciplinary care of patients with CKD. The specific review questions were:

- What clinical pharmacy practice related resources (structures, e.g. the multidisciplinary team, clinical pharmacy skill mix and time allocation) are in place and how are these matched to healthcare needs and demands to enable provision of care to chronic kidney disease (CKD) patients?

- What activities are performed (processes, e.g. medication review, prescribing) to care for patients with CKD, how and when are they performed?

- What are the outcomes of the structure and the processes on the effectiveness (Economic, Clinical, and Humanistic Outcomes (ECHO) model) [8] of care provided?

\section{Method}

\section{Data sources}

The systematic review protocol was registered with the International Prospective Register of Systematic Reviews (PROSPERO) (PROSPERO 2017 CRD42017065258). The protocol was constructed in accordance with PRISMAP (Preferred Reporting Items for Systematic review and Meta-Analysis Protocols) standards [9], and the review conducted and reported in accordance with PRISMA (Preferred 
Reporting Items for Systematic Review and Meta-Analysis) standards [10].

The Cochrane database was searched to identify any relevant systematic reviews. An electronic search of relevant databases (PubMed, International Pharmaceutical Abstracts (IPA), Cumulative Index to Nursing and Allied Health Literature (CINAHL), Medline and Scopus) was conducted from March 2010 to December 2018 thus providing an update on the review of Salgado et al. [7]. The search was carried out using Medical Subject Headings (MeSH) and other appropriate subject headings and text words. Scoping searches were conducted prior to finalising the search strategy. Boolean operators such as truncations (*), wild cards (\$), adjacent search options (e.g. adj2) were used where relevant. The following grouped terms were initially searched separately then in combination by two independent reviewers (FA \& SC). The primary search was conducted using the improved search strategy of the same terms as the original review as follows:

PubMed, IPA, CINAHL: ("pharmaceutical services" [MH+] OR "pharmacy" [MH+] OR "Pharmacies" [MH] OR "Pharmacists" [MH] OR "clinical pharmacist*" [TI/ AB/SU] OR "clinical pharmacy" [TI/AB/SU] OR "clinical pharmacies" [TI/AB/SU] OR "pharmacist*" [TI/AB/ SU] OR "pharmaceutical services" [TI/AB/SU] OR "pharmacies" [TI/AB/SU] OR "pharmacy" [TI/AB/SU]) AND ("kidney diseases" $[\mathrm{MH}+] \mathrm{OR}$ "renal replacement therapy" [MH+] OR "proteinuria” [MH+] OR “CKD” [TI/AB/SU] OR "nephropathy" [TI/AB/SU]).

Scopus:

("Pharmaceutical care" [TI/ABS/KEY] OR "Pharmacist" [TI/ABS/KEY] OR “Clinical pharmacy” [TI/ABS/KEY]) AND ("Chronic Kidney Disease" [TI/ABS/KEY] OR "Renal replacement Therapy" [TI/ABS/KEY] OR "Haemodialysis" [TI/ABS/KEY] OR "Kidney failure" [TI/ABS/KEY]). The bibliography list of included studies was reviewed to further identify additional references.

\section{Study selection and data extraction}

Only quantitative studies (randomised and non-randomised controlled and uncontrolled trials, cohort studies and before and after evaluations) published in peer-reviewed journals were included in the review. Papers published in English and focusing on researching clinical pharmacy practice and the role of the pharmacist in managing patients with CKD were included. Studies not addressing the topic, literature based only on conceptual models, i.e. lacking empirical evidence, grey literature including conference proceedings, abstracts and unpublished studies were excluded. Observational studies were excluded since they did not address the aim of this review.
Title and abstract screening and quality assessment for inclusion were conducted independently by two reviewers (FA and SC), with any disagreements resolved by discussion with a third independent reviewer (DS).

\section{Quality assessment}

An independent, duplicate quality assessment of each study was undertaken (DS, TJ, FA \& SC). All controlled, uncontrolled and descriptive studies were assessed using the mixed methods appraisal tool (MMAT), a validated and unique tool for appraising different types of study designs [11]. All controlled studies included in this review were additionally assessed for quality using the Downs and Black's method in line with the original review [12], a validated tool with a scoring scale consisting of 27 questions grouped into five domains (reporting, external validity, bias, confounding and power). The total score is 32 and is expressed as rates, the higher the score the better the quality of the paper in terms of methodology (maximum is 1) [12]. To classify scores, the approach of Machado et al. was applied [13] (i.e. < 0.5 was considered 'weak', $0.5-0.69$ were 'fair', 0.7-0.79 'good' and 0.8-1.0 'very good').

\section{Data extraction}

Data extracted included: primary author, year of publication, aim/objectives, design, duration, setting, participants, pharmacist interventions, key findings or main outcomes and conclusion. Structures, processes and outcomes were adapted from Donabedian's quality of care model [14]. Structure was defined as the 'resources required for the pharmacist to be able to provide care to renal patients such as requiring special training, availability of policies and procedures for practice etc'. Process was defined as 'the activities that are performed by the pharmacist on a daily basis or on specific intervals and how and when they are performed. These activities may include: daily clinical rounds, involvement in patients' management plans, medication reviews, therapeutic recommendations and pharmacist prescribing. Outcome measures included clinical outcomes such as: clinical parameters, medication-related adverse events, mortality and morbidities, humanistic outcomes such as: quality of life and economic outcomes such as: rate of hospitalisation and cost of inappropriate therapies. In addition, pharmacists' intervention was defined in the previous review as "any action with the aim of modifying the process of use of drugs, either in patients' activities or in medical or health care practitioners' activities" [7]. 


\section{Data synthesis}

Due to heterogeneity in the data obtained from the included papers (type of patients, study design, outcomes measured), only descriptive and narrative synthesis was possible. All findings were considered by two independent reviewers to ensure robustness and consistency in execution of the review process.

\section{Results}

\section{Study selection and data extraction}

No systematic reviews were identified from the Cochrane database and no additional primary studies were identified from the bibliography lists of included studies.

Databases searches identified 4140 potential articles to screen further for eligibility (Fig. 1). Only 47 articles met the inclusion criteria and after quality assessment were of a standard deemed acceptable for inclusion in the review.

\section{Quality assessment}

The Downs and Black's mean score of the 20 controlled studies was $0.557(\mathrm{SD}=0.075)$. All papers presented 'fair' quality with the exception of four that scored $<0.5$ and was therefore considered 'weak' quality. The quality assessment of all the included studies using the MMAT tool for the randomised $(n=10)$, non-randomised $(n=20)$ and descriptive studies $(n=17)$ are shown in Figs. 2, 3 and 4.

\section{Data extraction}

Tables 1 and 2 detail the data extraction characteristics of controlled and uncontrolled studies included in the systematic review [15-61].
Fig. 1 PRISMA Chart describing study retrieval and selection

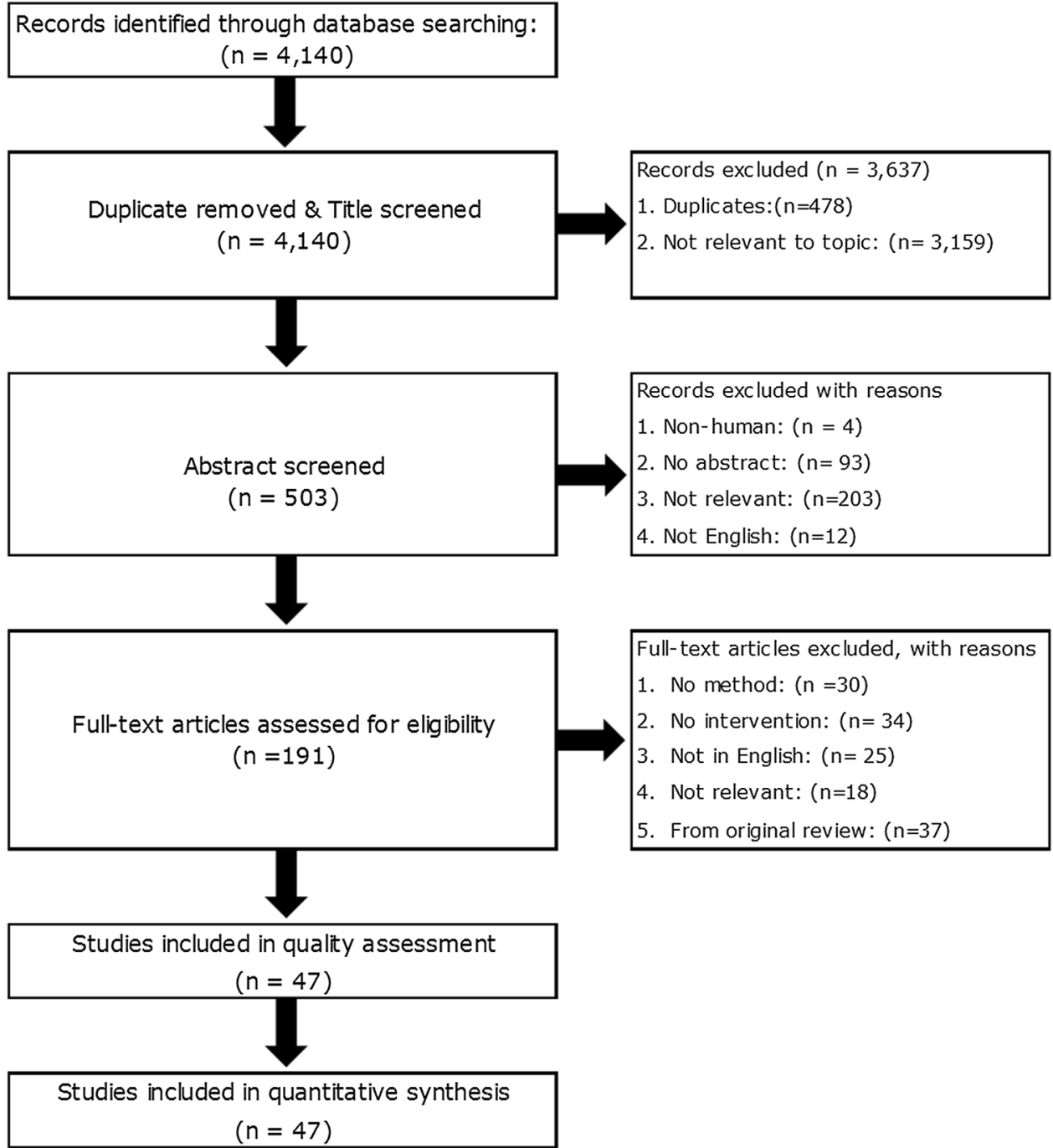




1. Are there clear quantitative research questions or objectives?
2. Do the collected data allow address the research question or
objectives?
3. Are participants (organizations) recruited in a way that
minimizes selection bias?
4. Are measurements appropriate regarding the
exposure/intervention and outcomes?
5. In the groups being compared, are the participants
comparable?
6re there complete outcome data (80\% or above), or
acceptable response rate $(60 \%$ or above)?

Fig. 2 Stacked bar chart representing quality of quantitative Randomized Controlled Trials $(n=10)$. The $\%$ values above represents the proportion for each response as agreed between reviewers for the papers included for each study design

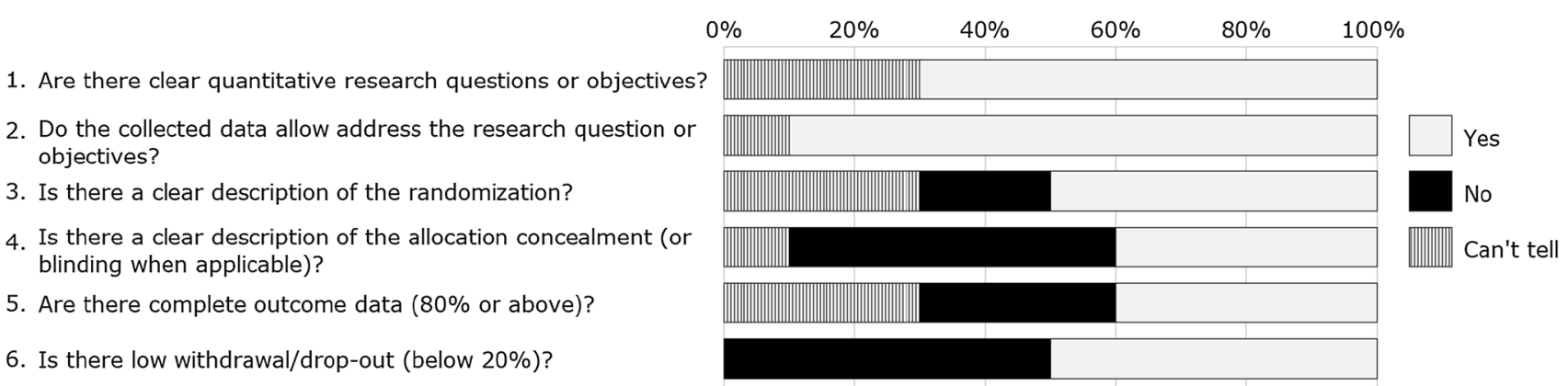

Fig. 3 Stacked bar chart representing quality of quantitative non-randomized studies $(n=20)$. The $\%$ values above represents the proportion for each response as agreed between reviewers for the papers included for each study design

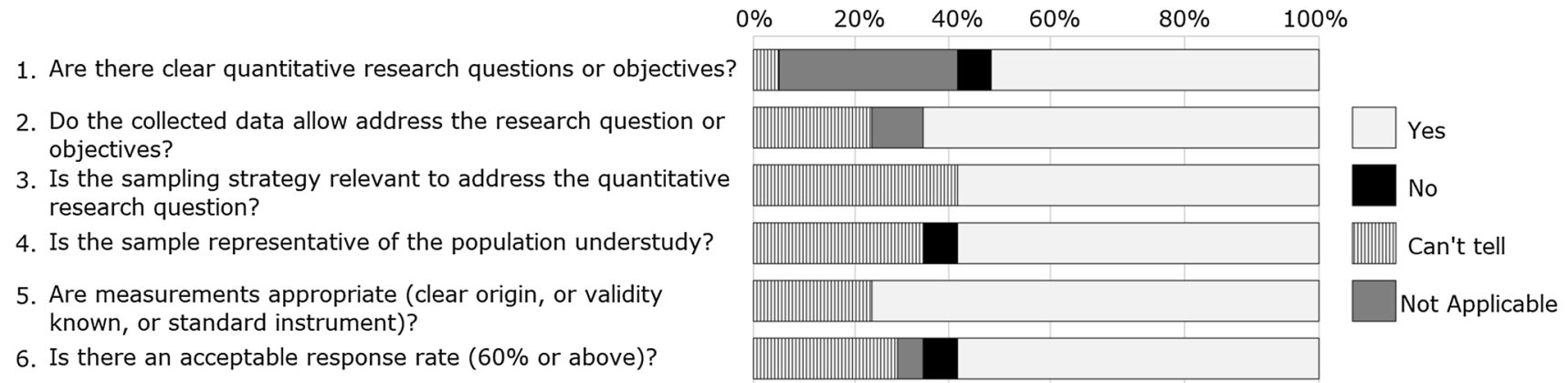

Fig. 4 Stacked bar chart representing quality of quantitative descriptive studies $(n=17)$. The $\%$ values above represents the proportion for each response as agreed between reviewers for the papers included for each study design

\section{Study characteristics}

The 47 studies were carried out in a variety of geographic locations: USA $(n=10)$, Iran $(n=5)$, India $(n=7)$, France $(n=3)$, Spain $(n=3)$, Jordan $(n=2)$, China $(n=2)$, Japan $(n=3)$, Singapore $(n=2)$, Nigeria, Taiwan, Australia, Saudi Arabia, Germany, Netherlands, Indonesia, Norway, Canada and the UK ( $n=1$ in each country). Two studies from 2008 to 2009 were not included in the systematic review of Salgado et al. [7], hence were considered as part of this review.
Thirty-one studies were conducted in hospital settings (wards, intensive care units (ICU), clinics, departments and dialysis units) and 16 in primary care settings, including clinics and community pharmacies. The follow-up time in all included papers ranged from 4 weeks to 24 months with a mean of 9.4 (standard deviation, $\mathrm{SD}=5.08$ ) months, with four studies with unclear duration.

The majority of studies $(n=27)$ used an uncontrolled study design, 21 prospective and six retrospective. The remaining 20 were controlled, ten of which were randomised 


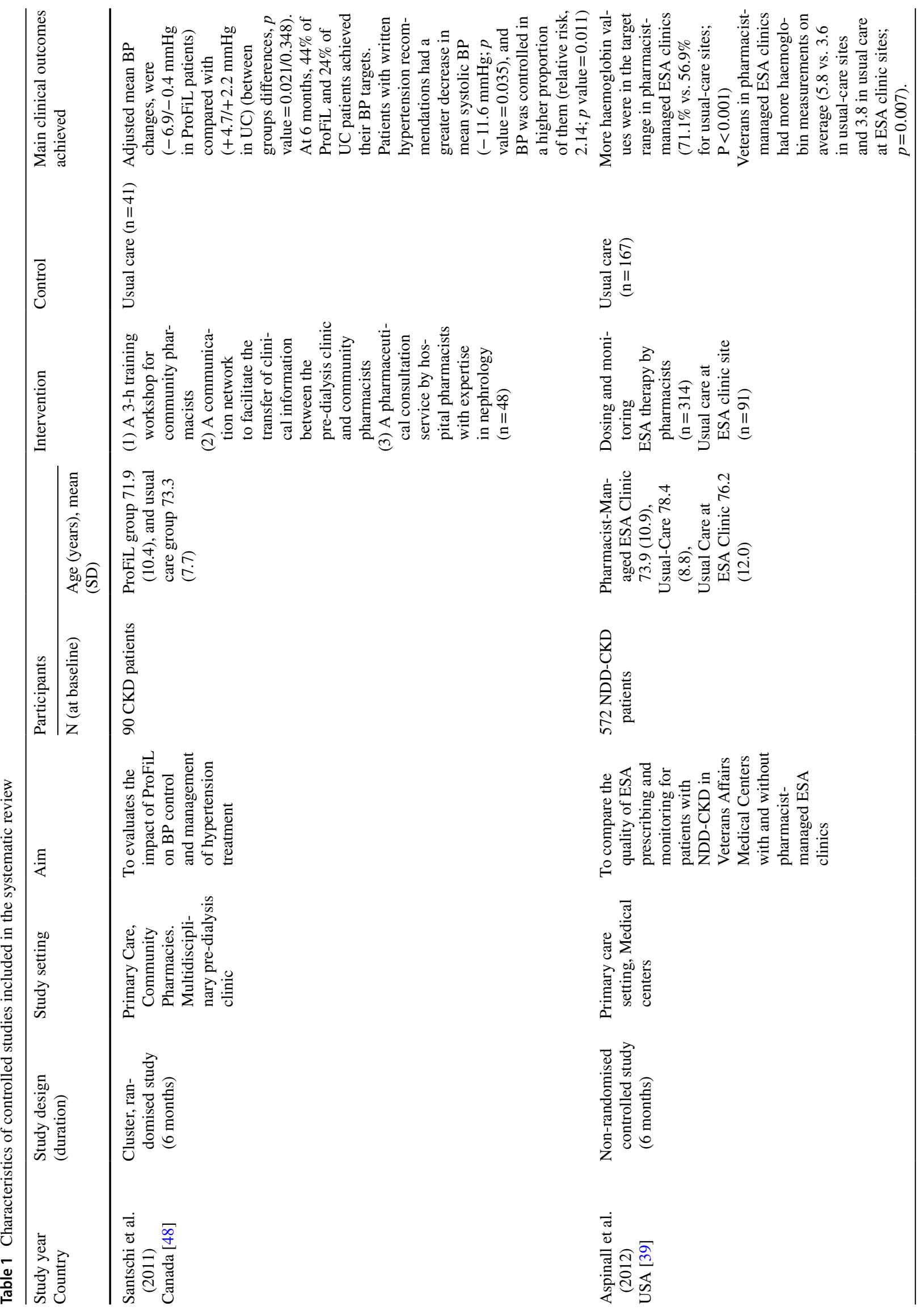




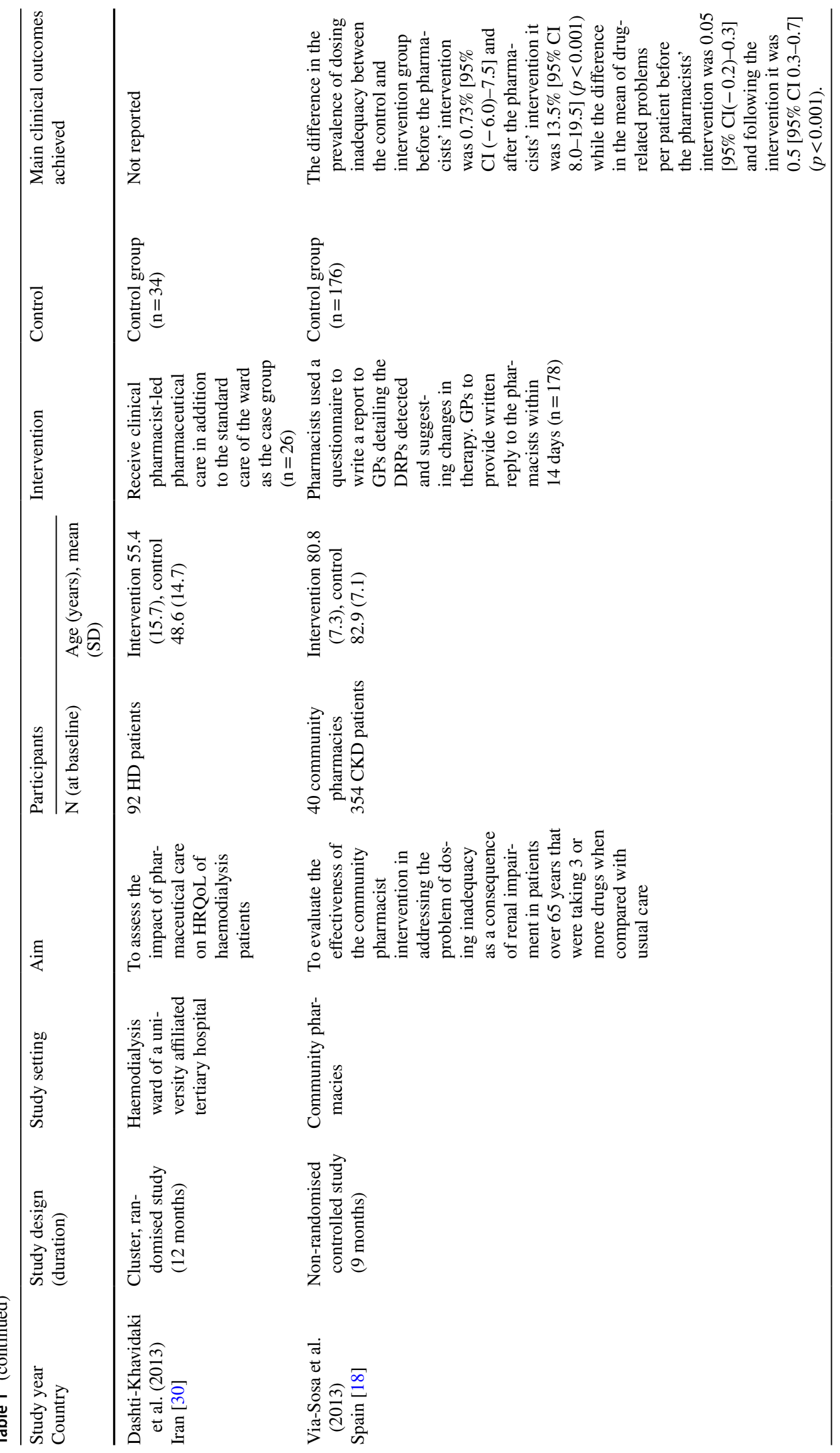




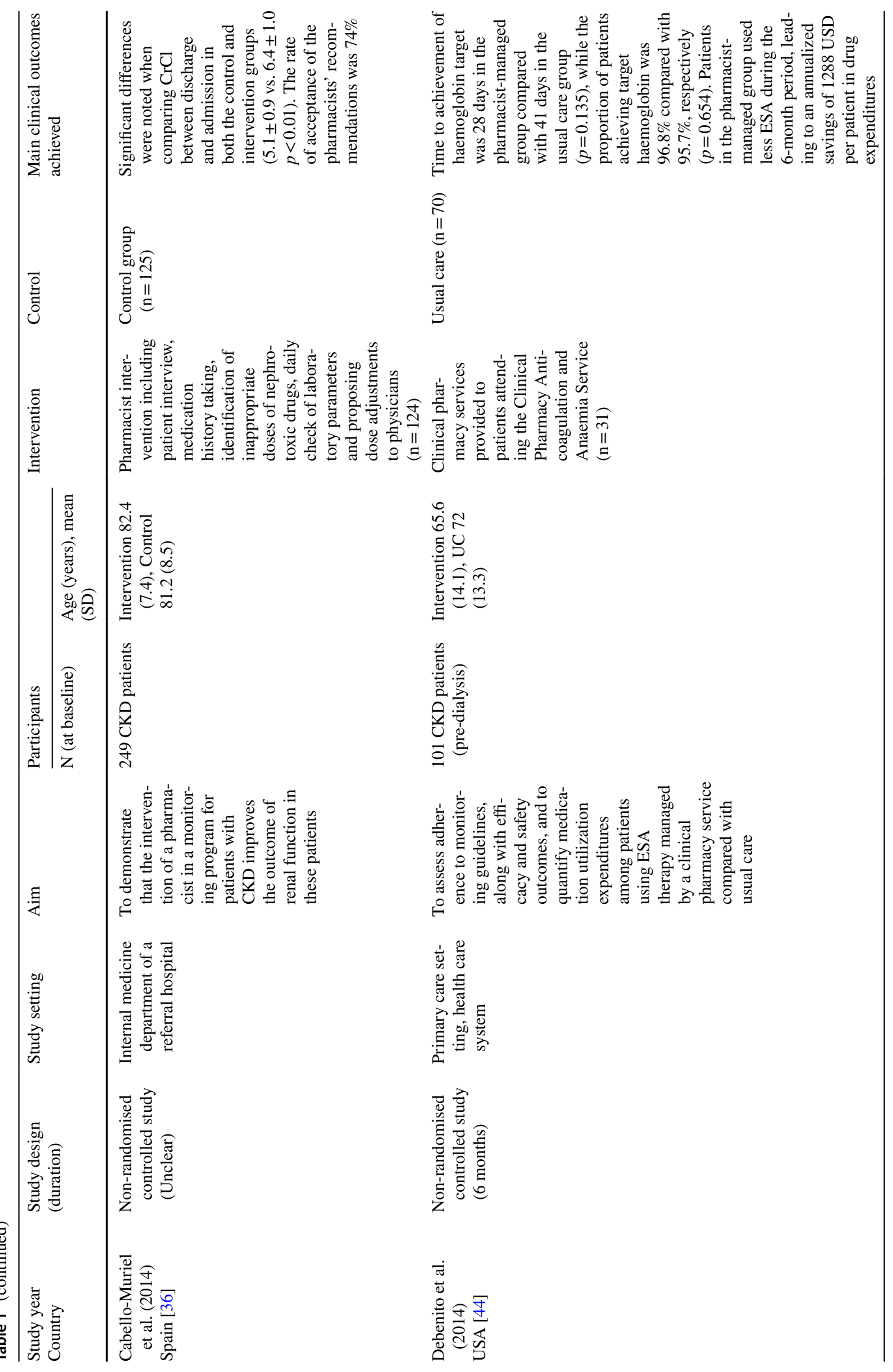




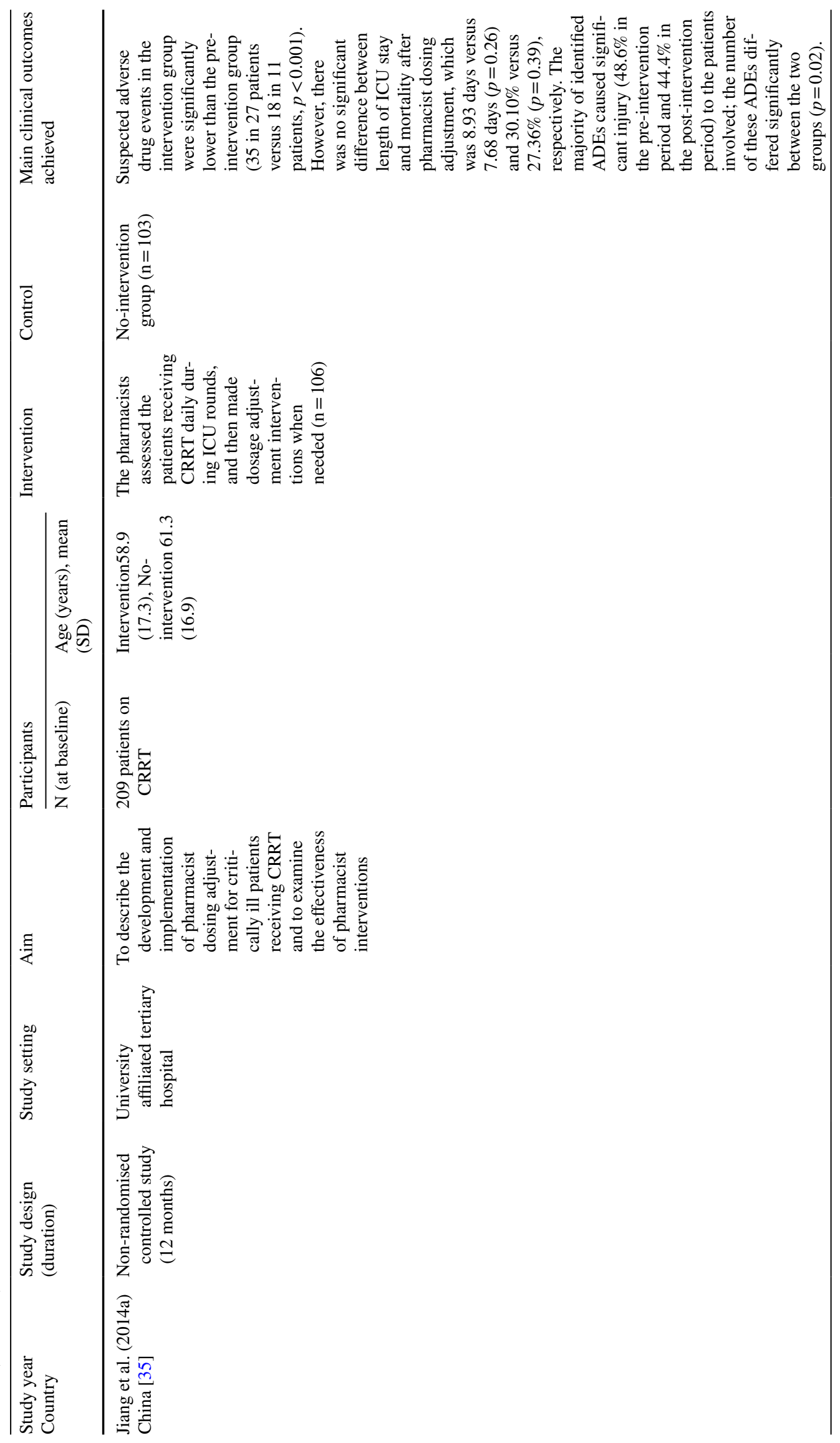




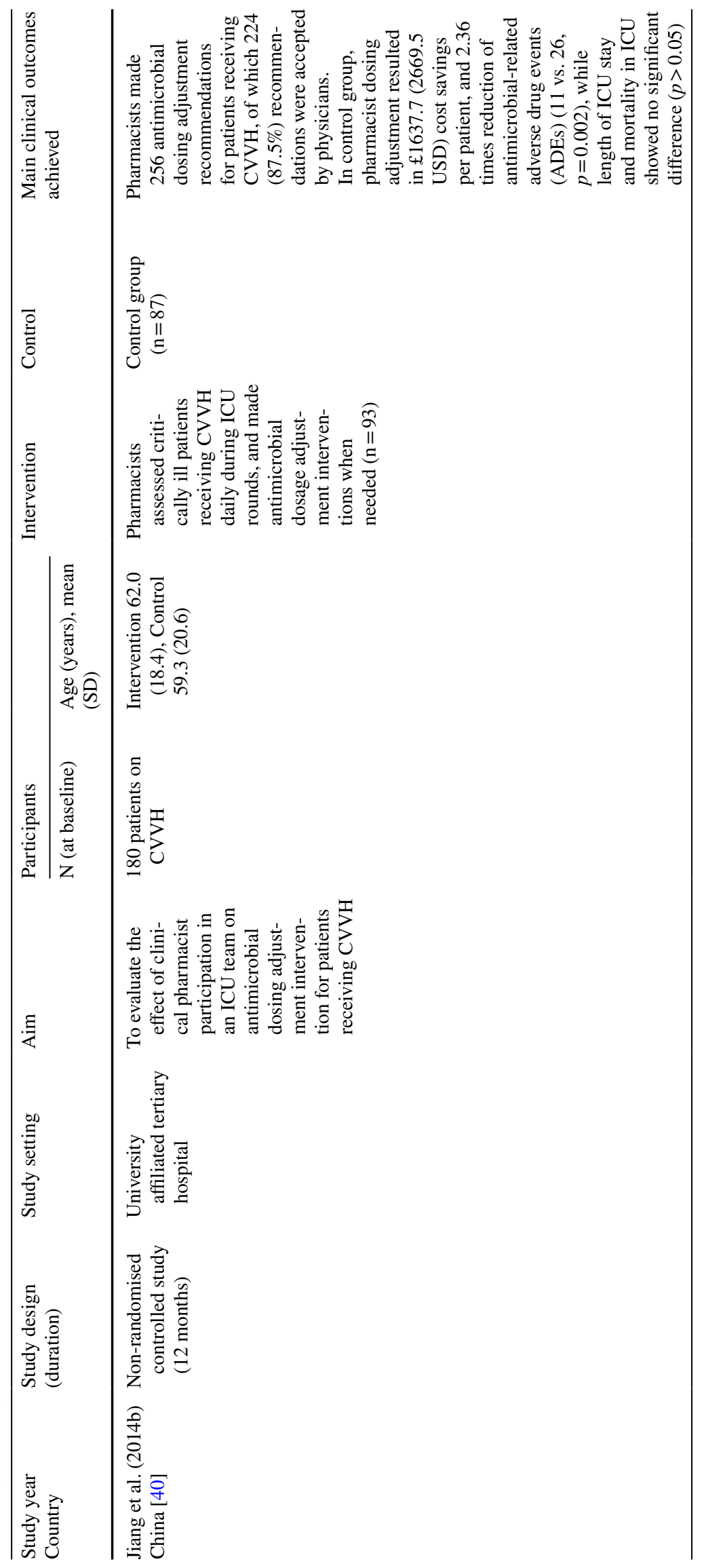




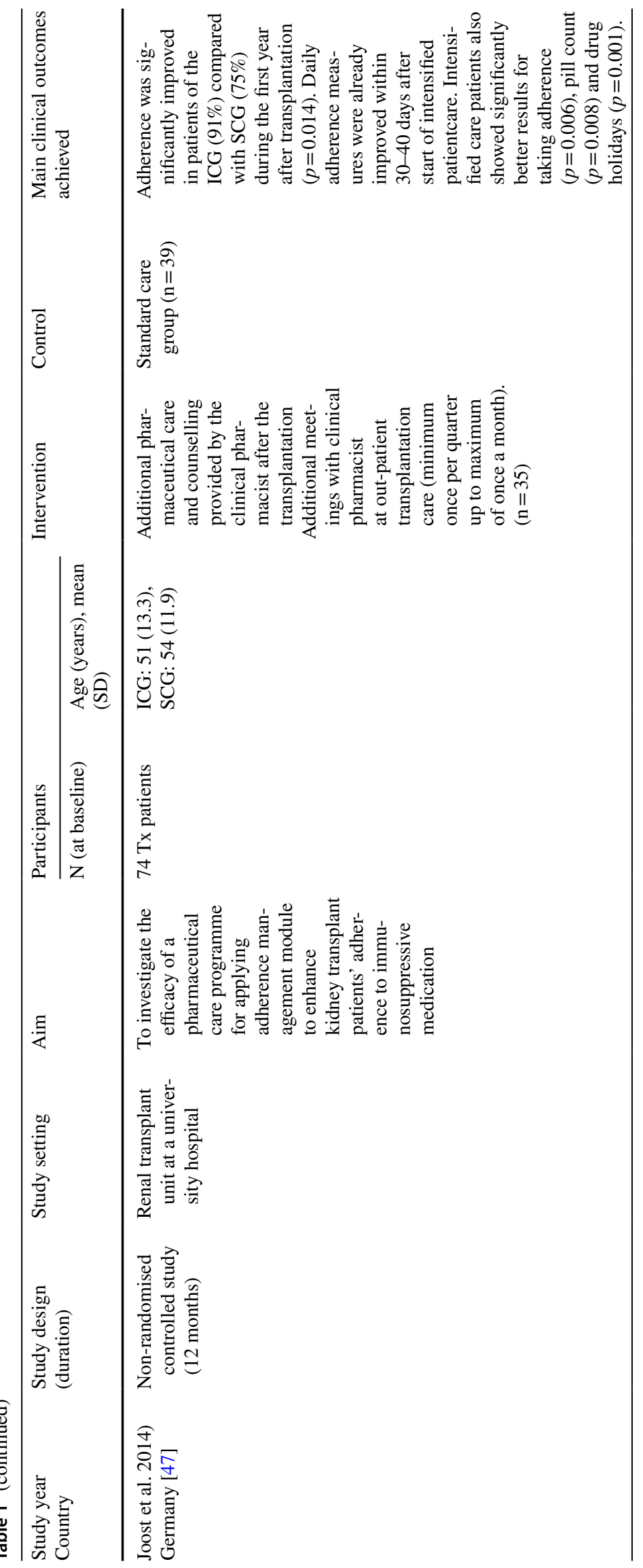

Springer 


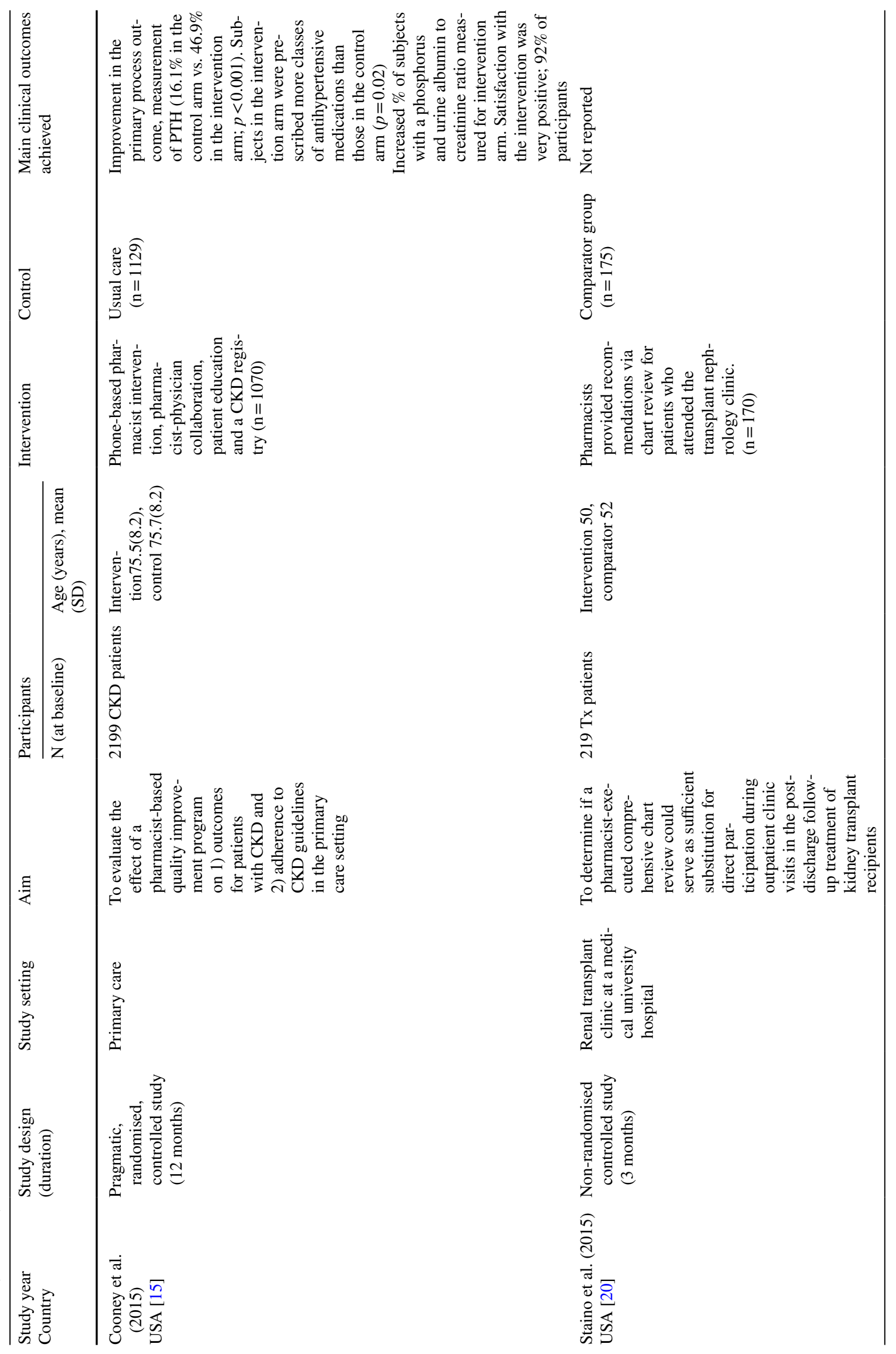




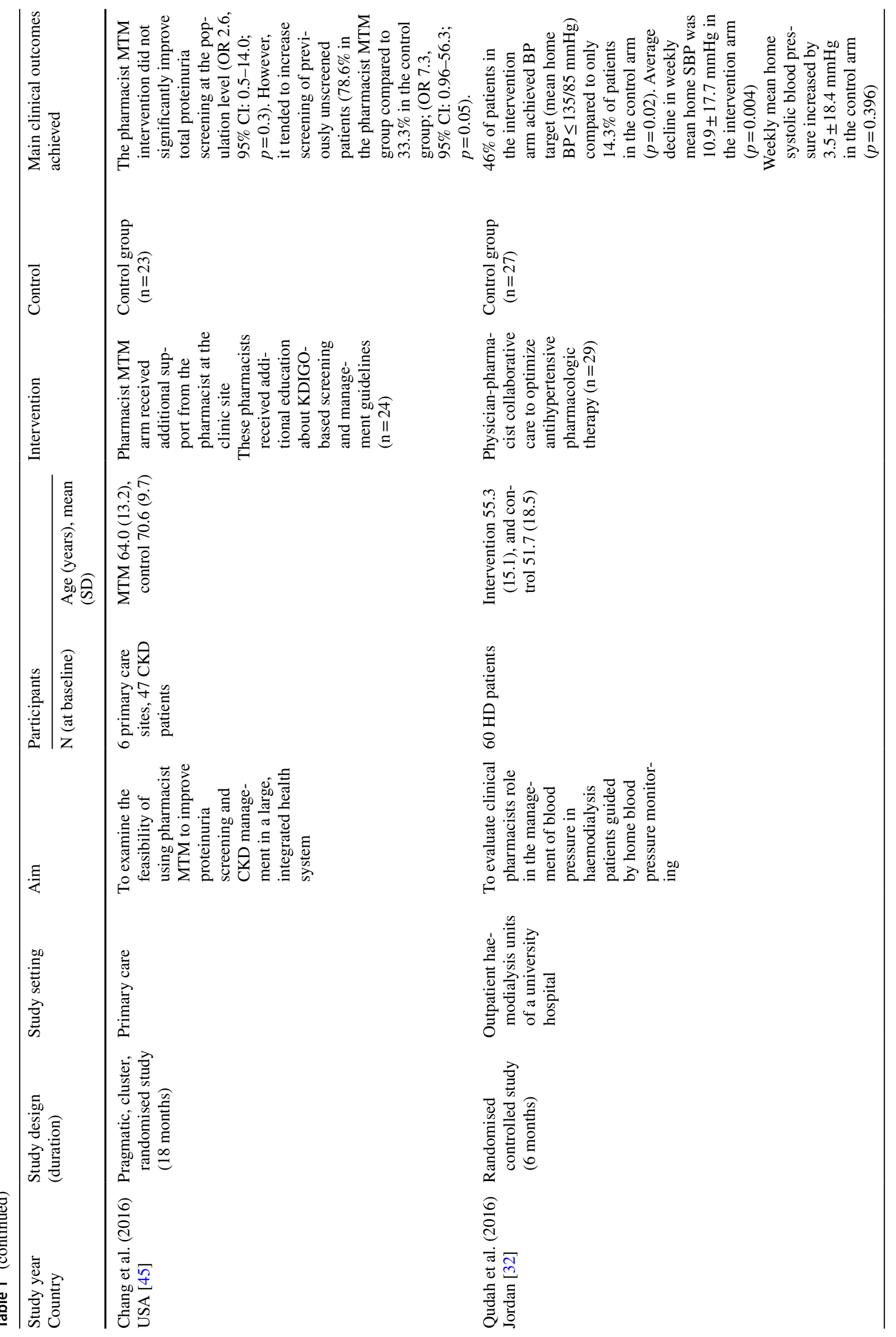




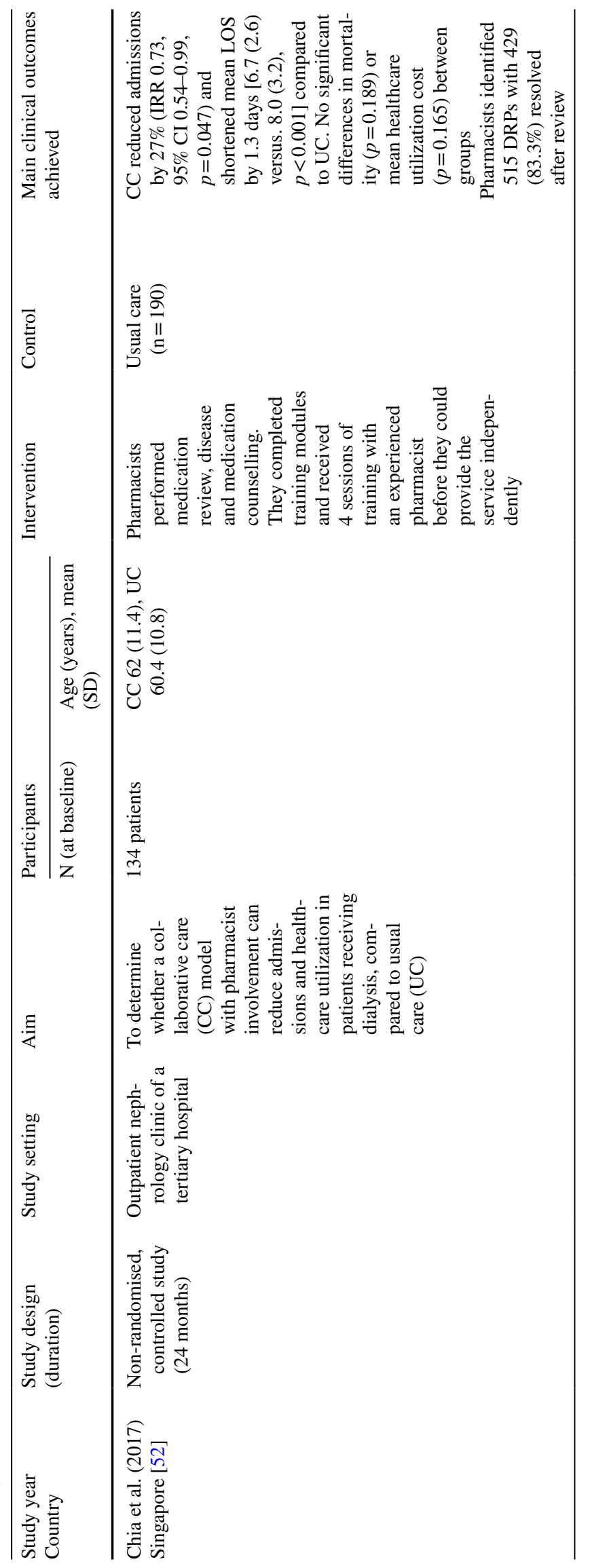




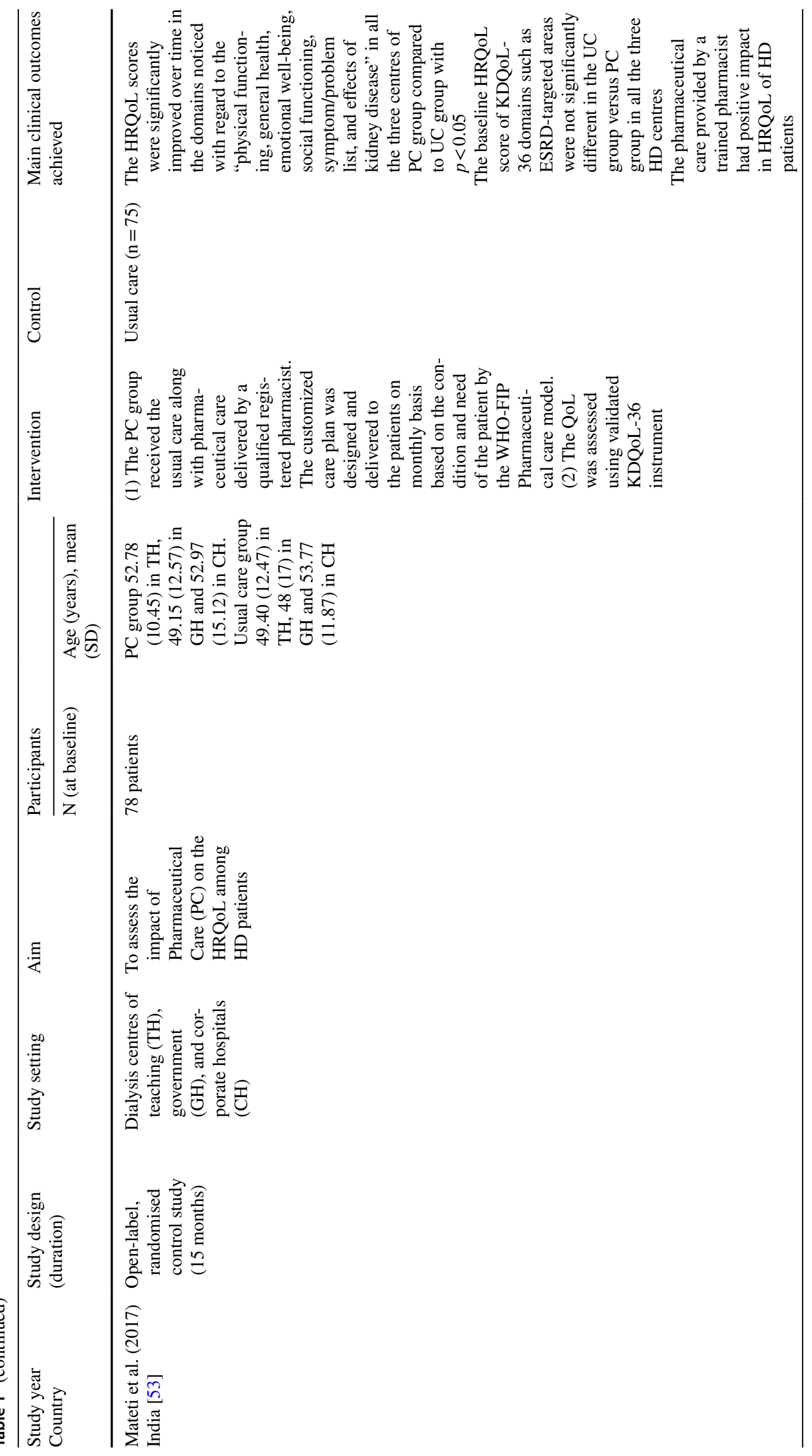




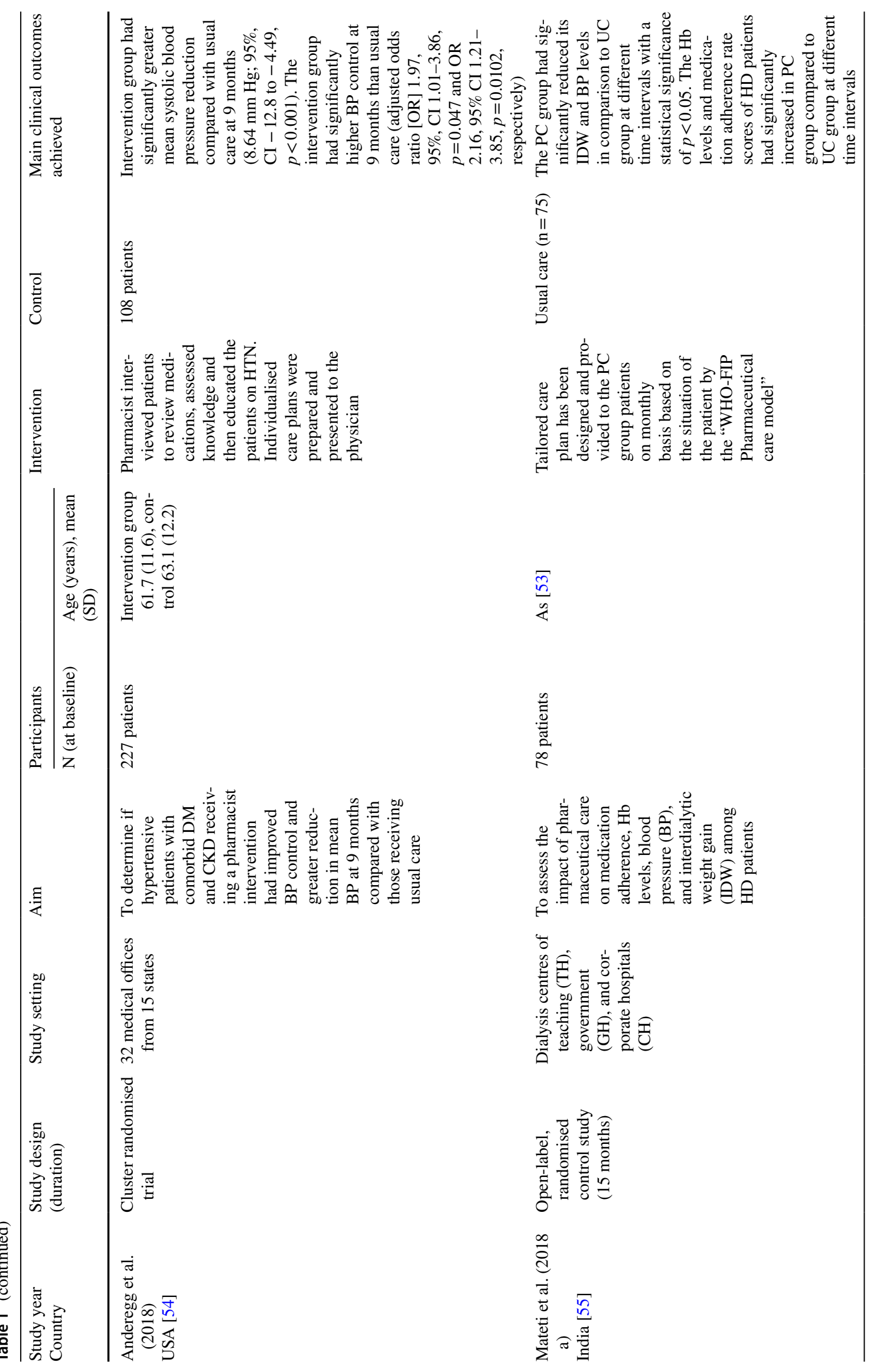




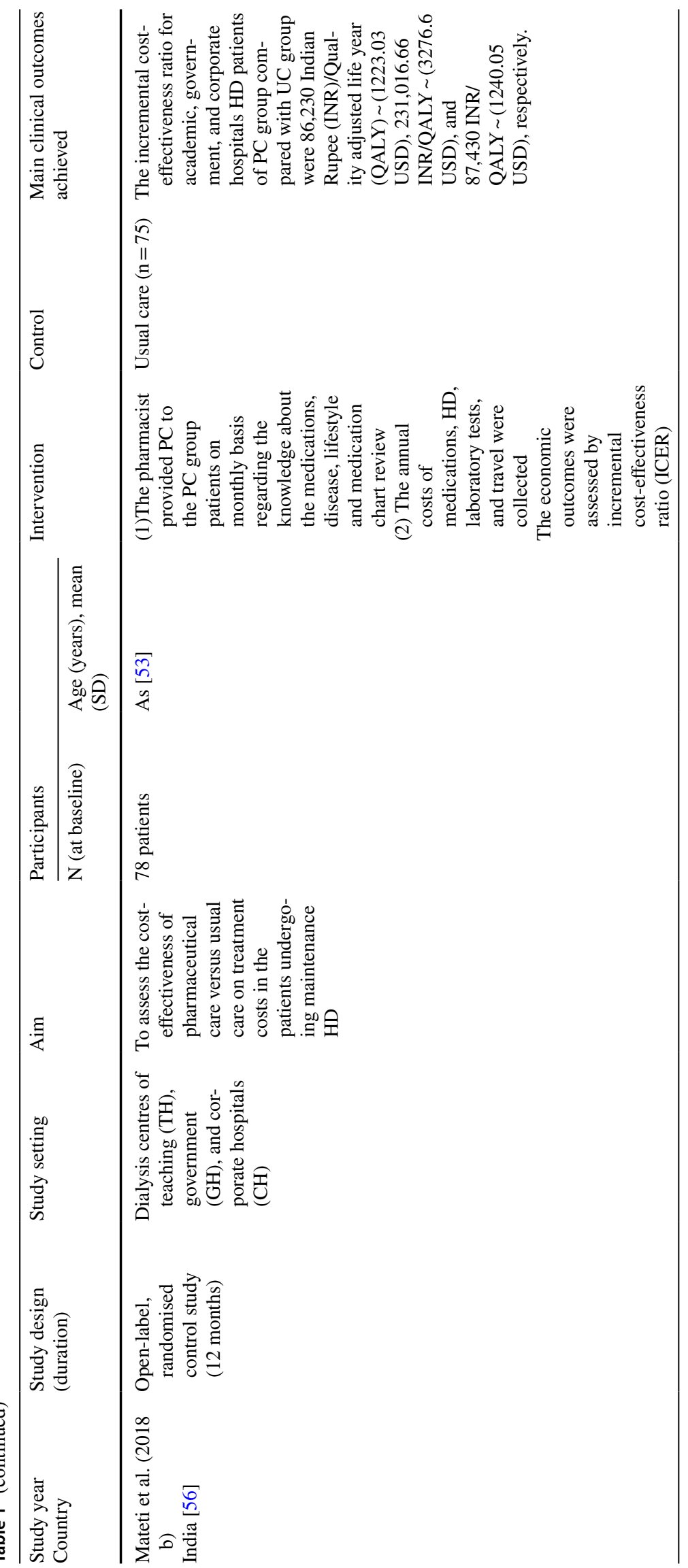




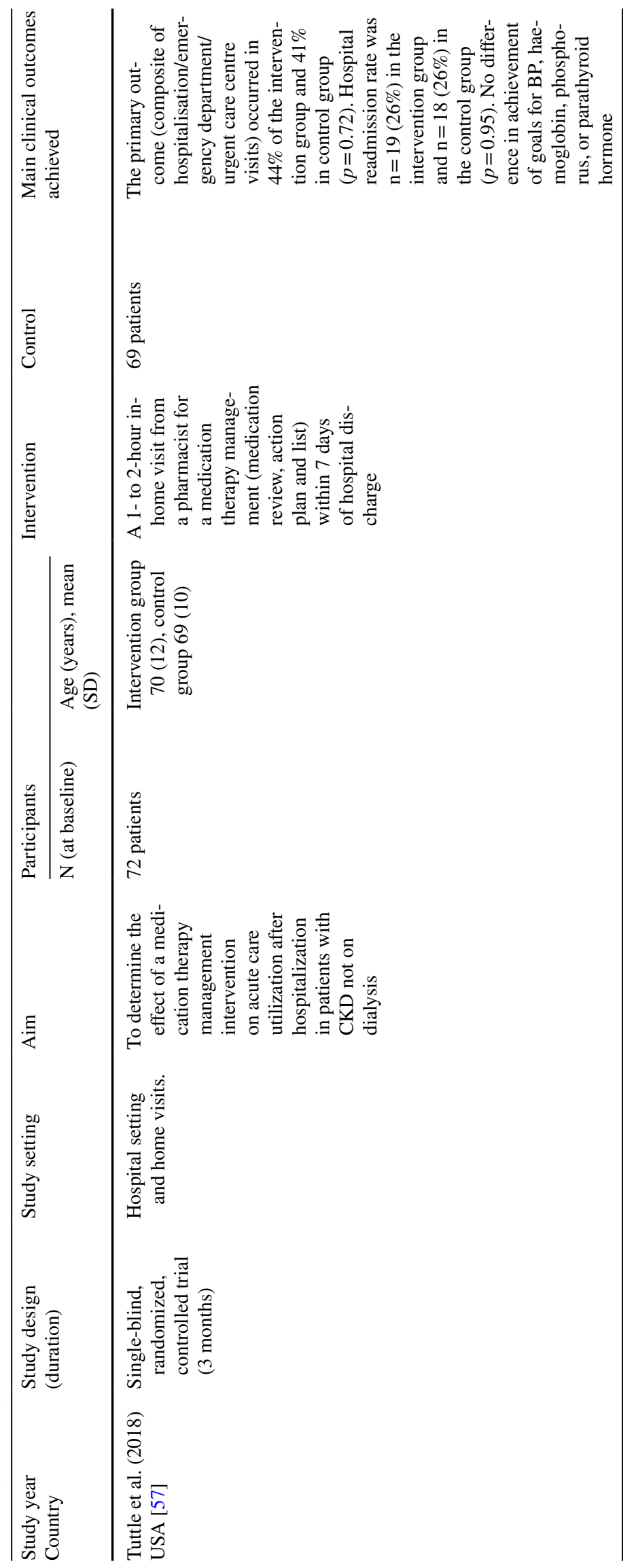




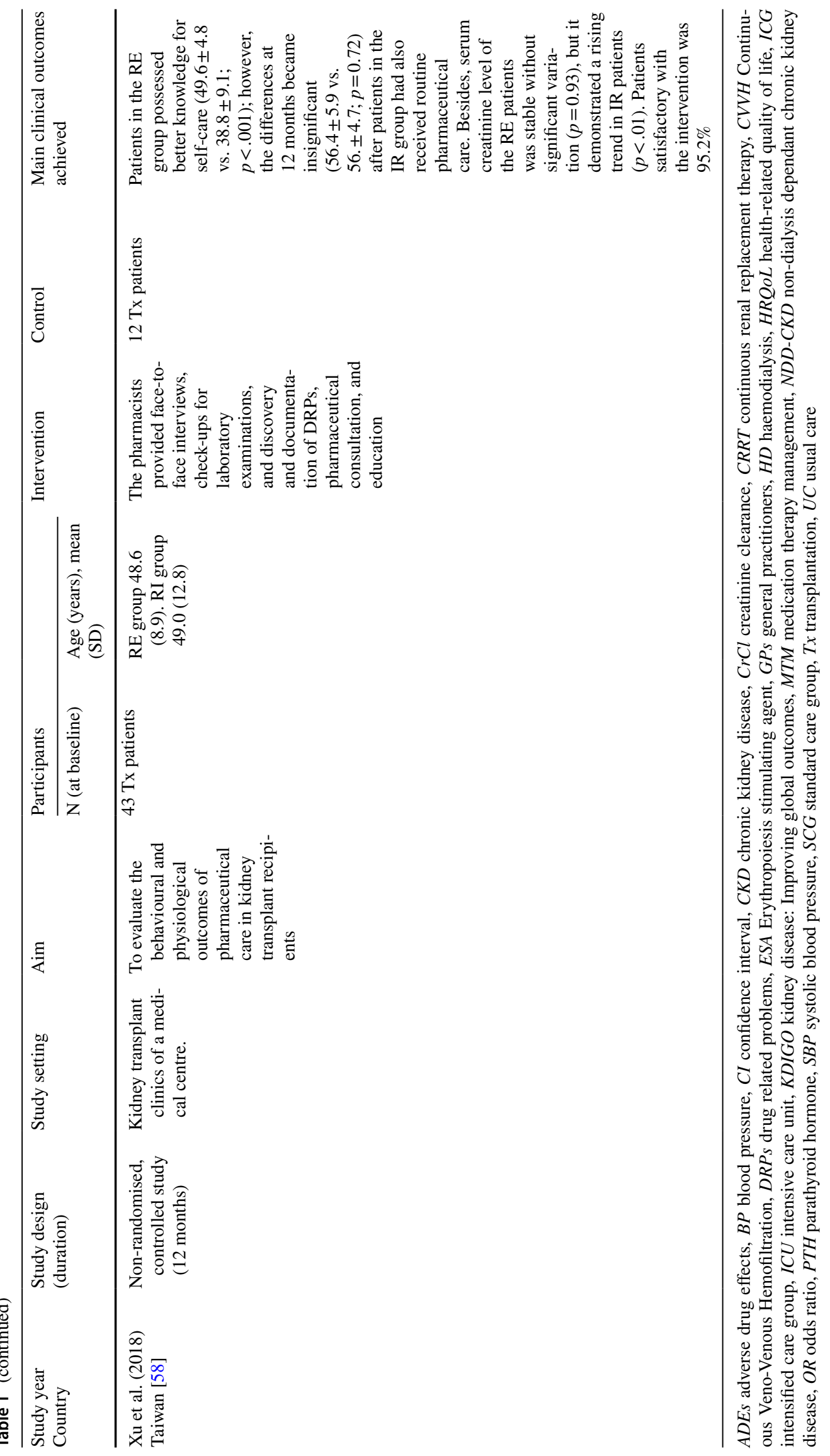




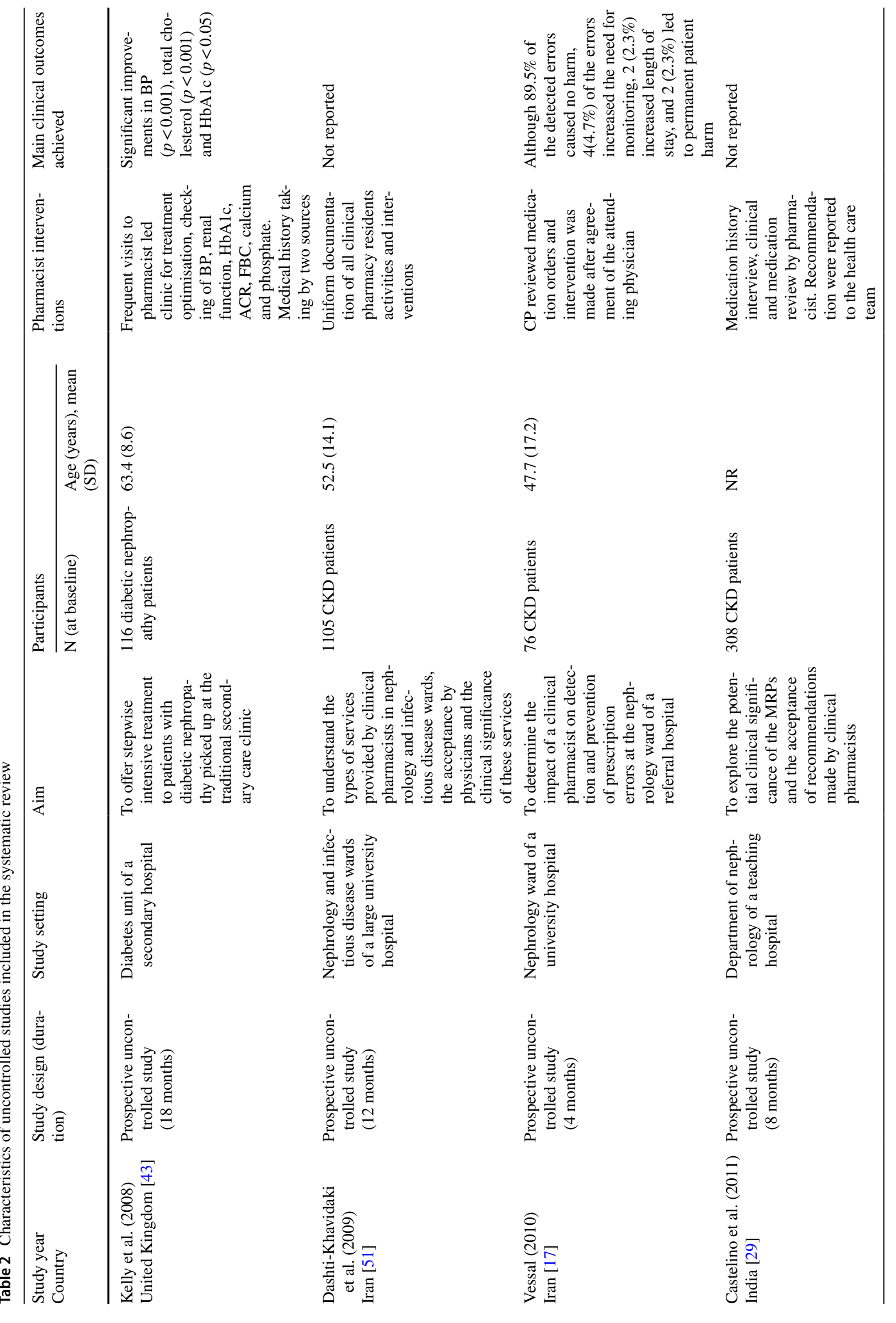




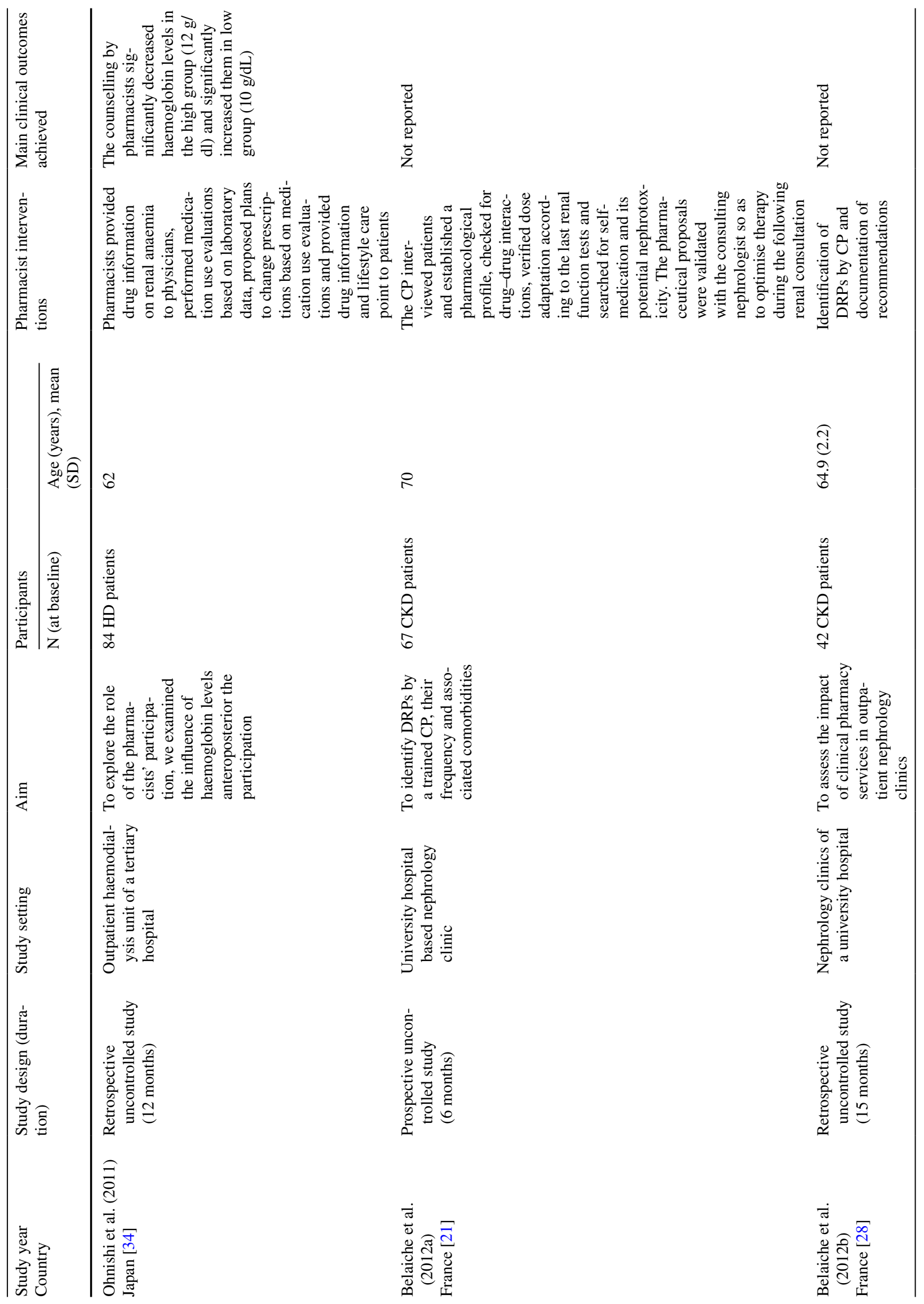




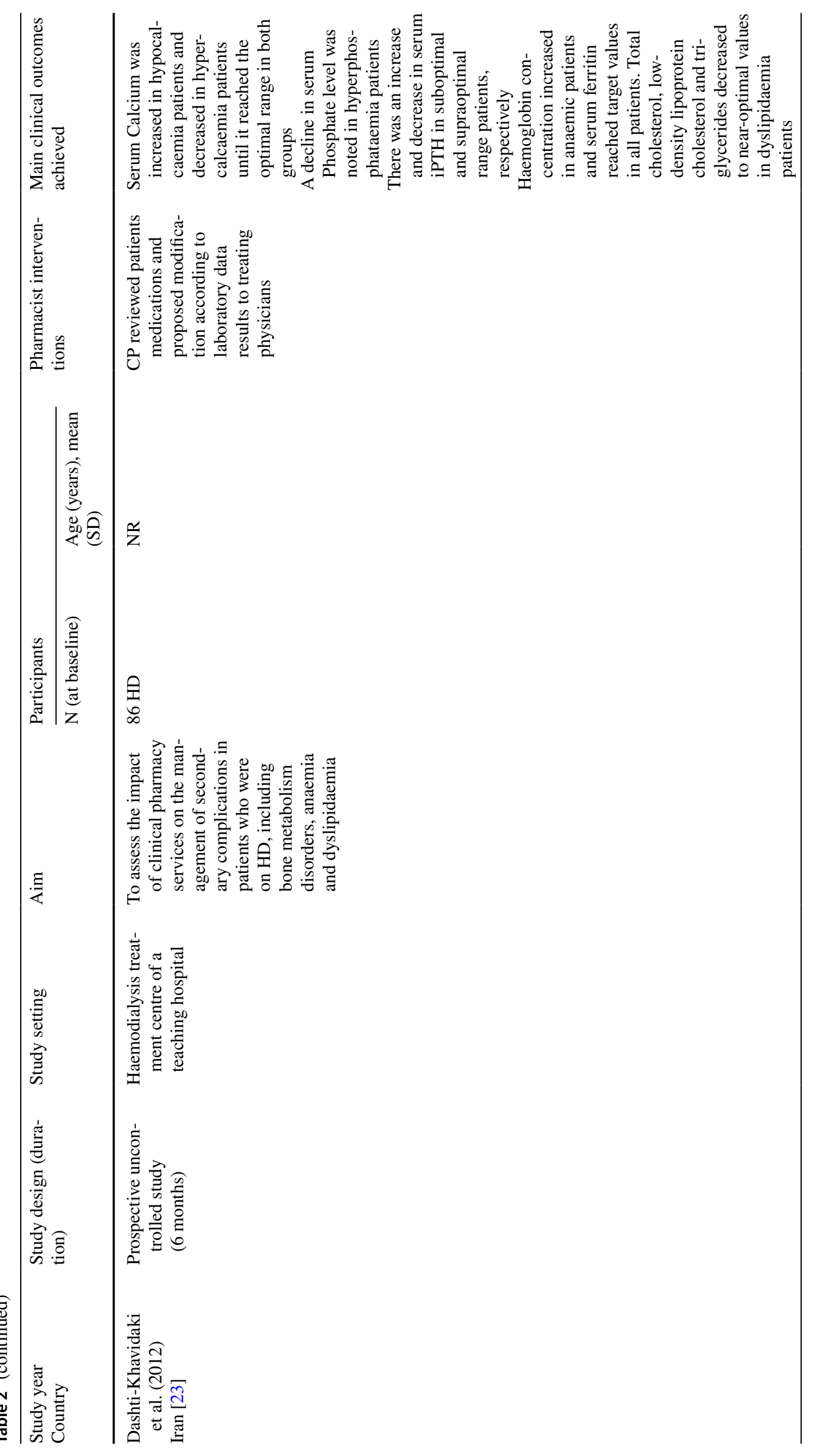




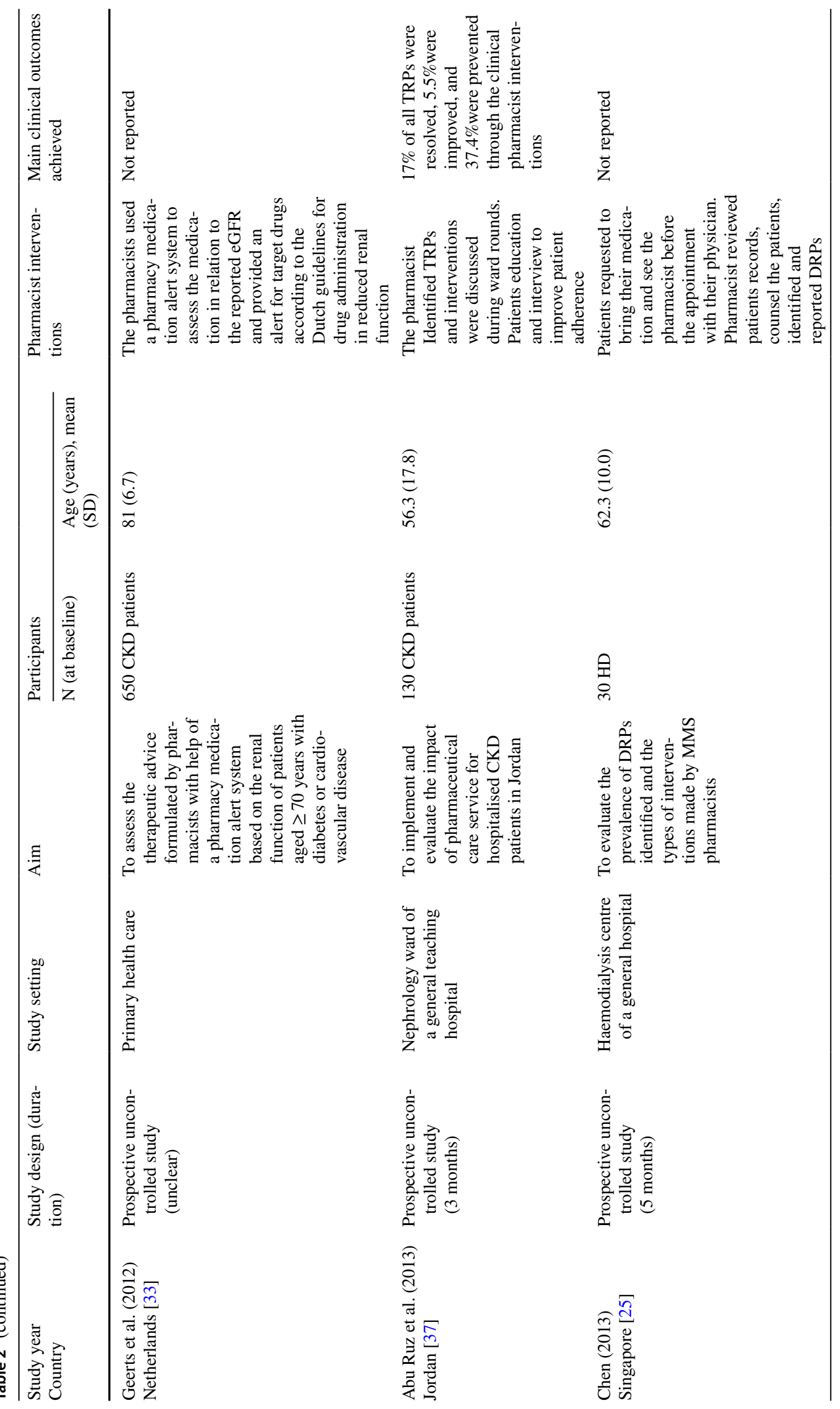




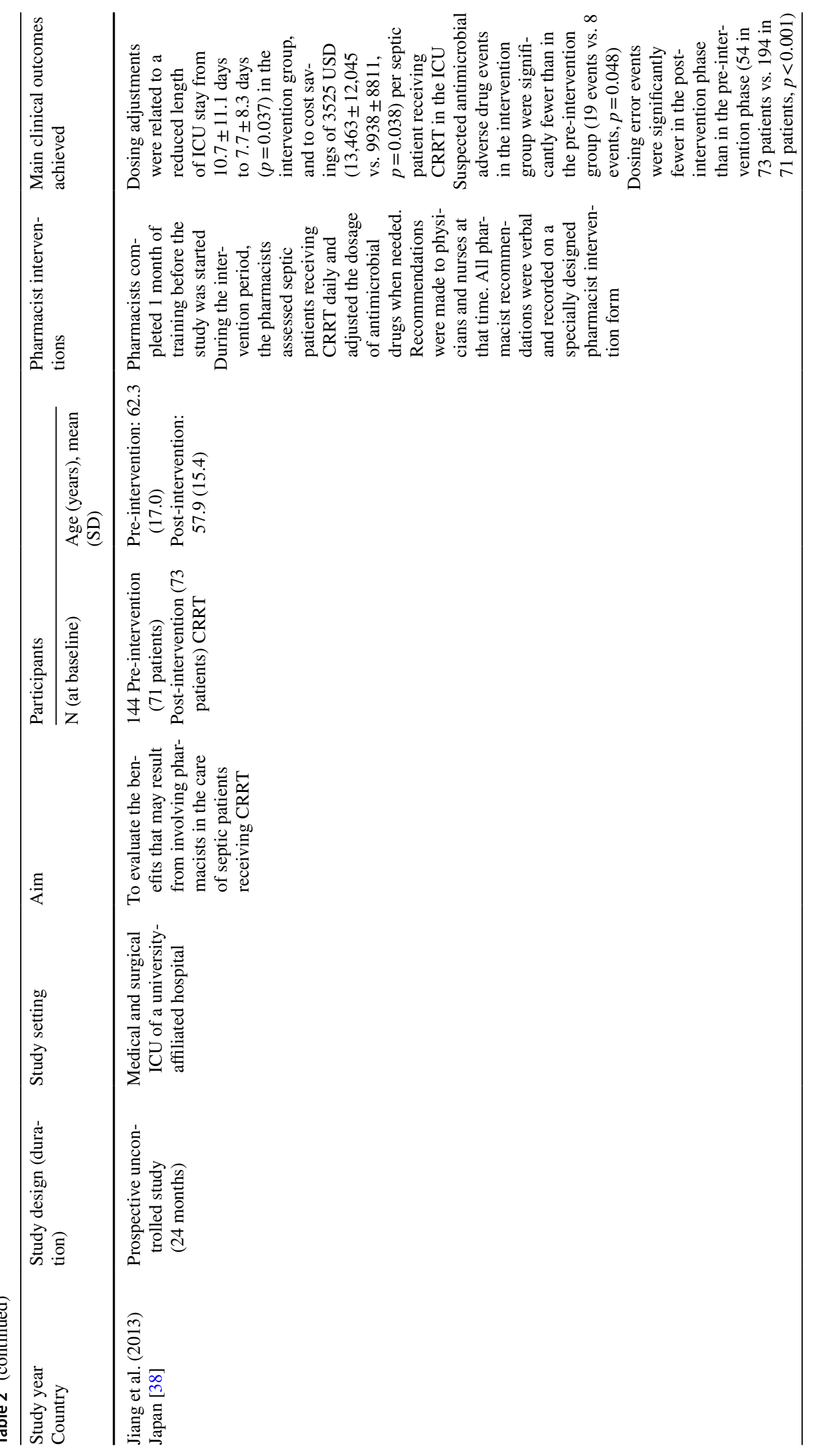




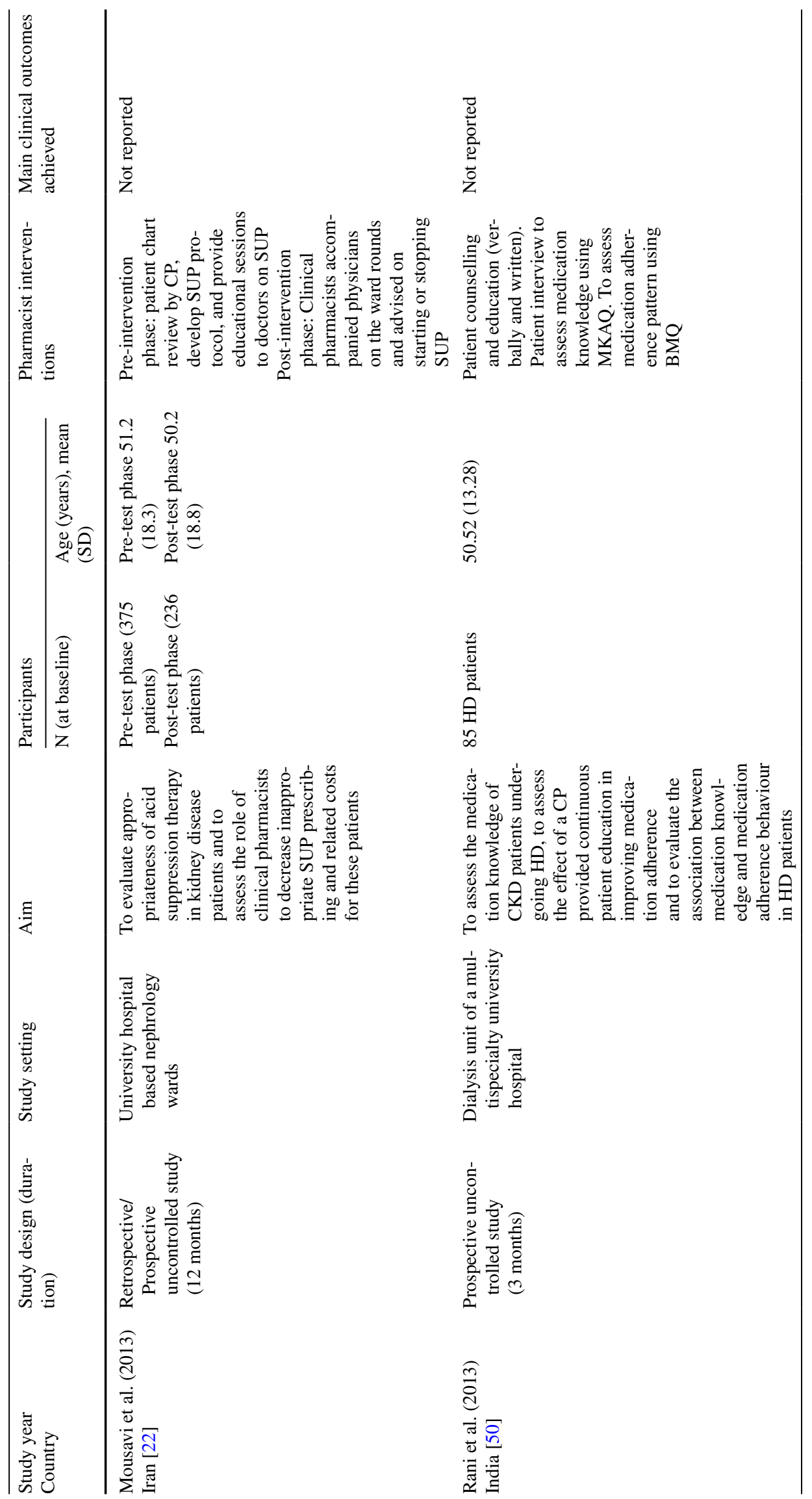




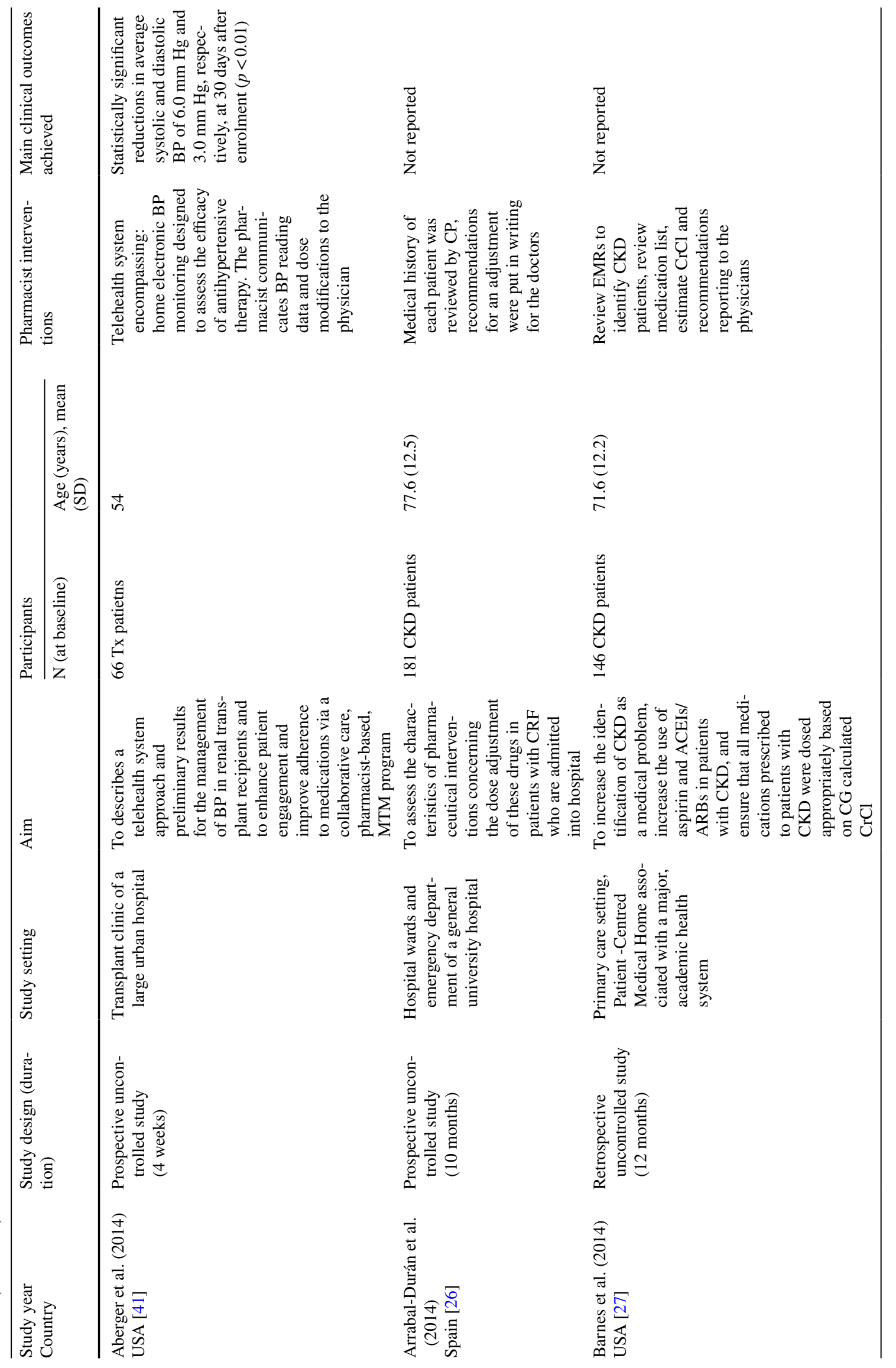




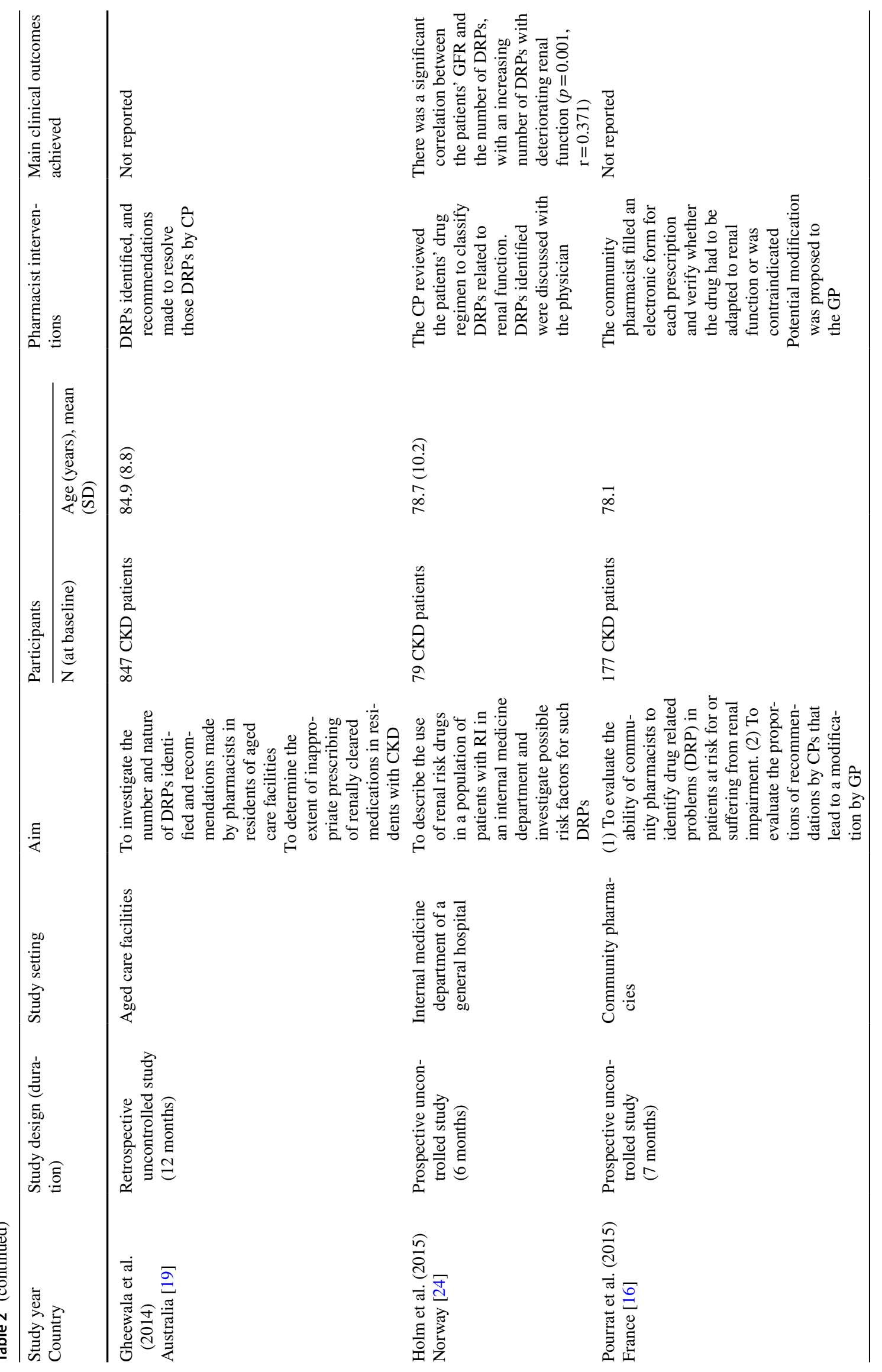




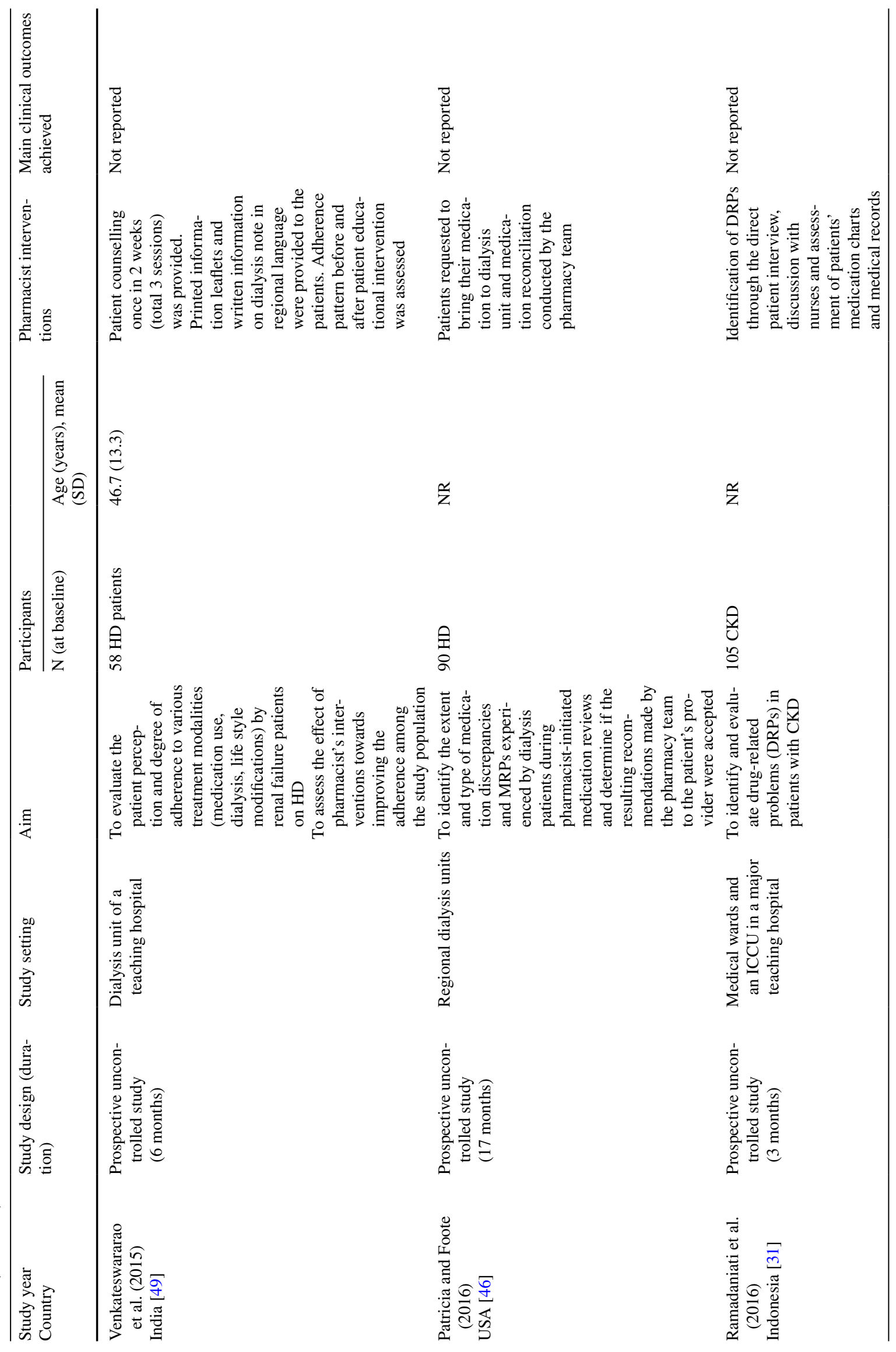




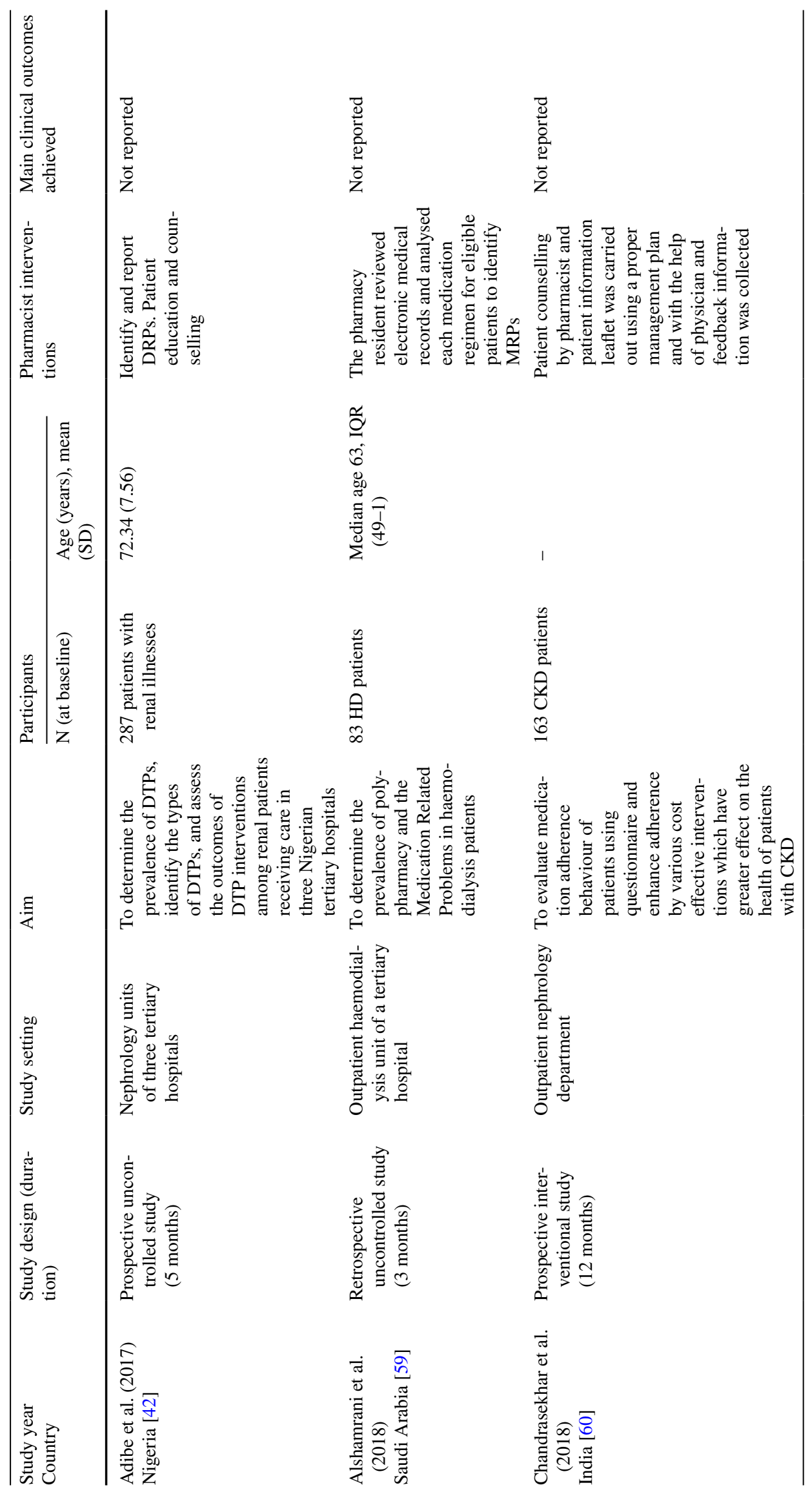




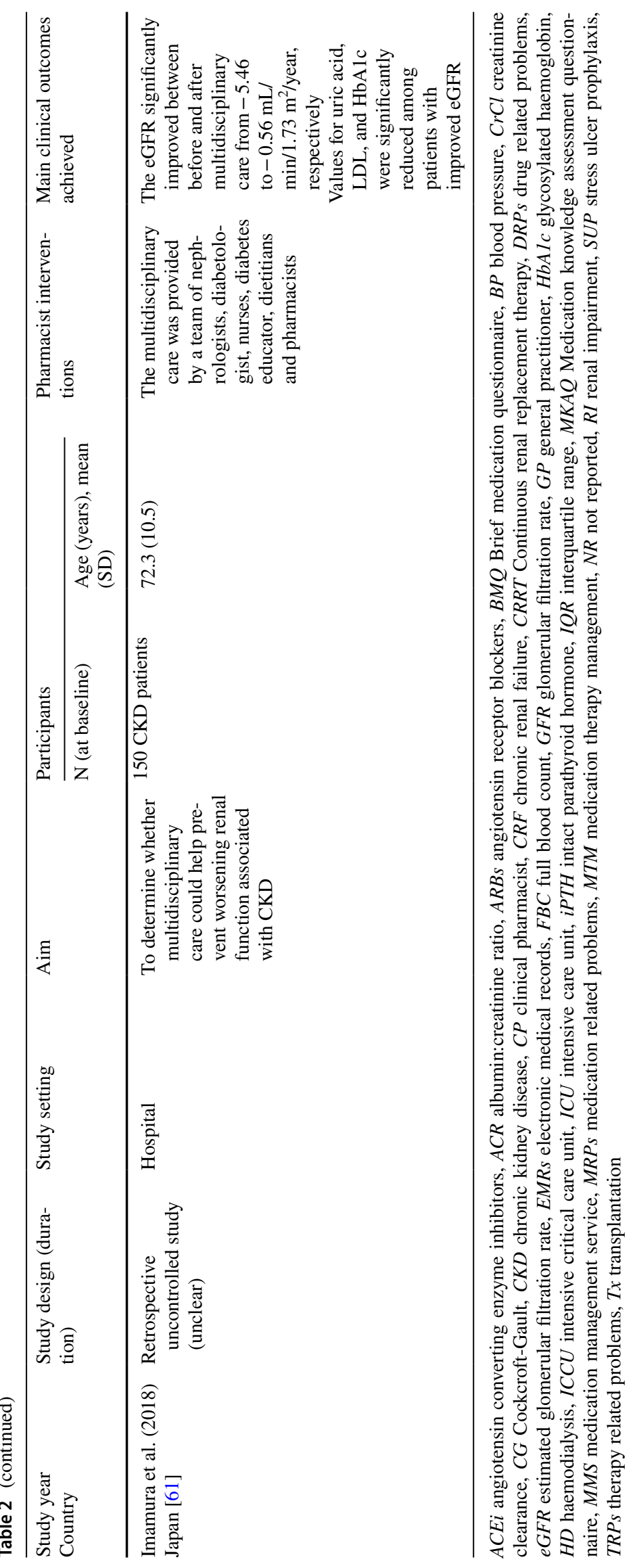


and ten non-randomised. According to Thomson Reuters Journal Citation Report at the time of publication the median impact factor of the journals of articles included was 1.348 (IQR 0.52-2.01), $\mathrm{n}=45$, two journals did not have an impact factor at the time of publication.

Patient mean age was 46.7-84.9 years, with five studies failing to report age $[23,29,31,46,60]$. Of the total of 11,122 patients from all studies, 9151 were at various stages of chronic kidney disease not on dialysis, 1036 were haemodialysis (HD) dependent, 533 receiving other forms of renal replacement therapies such as continuous renal replacement therapy (CRRT) or continuous veno-venous hemofiltration (CVVH), and 402 were transplant patients.

Outcomes were reported in 37 papers, with 25 of these (67.6\%) also reporting details of the processes of care, and four $(10.8 \%)$ reporting structures, processes and outcomes. Outcomes reported were: clinical only $(17,45.9 \%)$, economic with linked clinical $(5,13.5 \%)$, humanistic with linked clinical $(4,10.8 \%)$, humanistic only $(2,5.4 \%)$ and economic only $(2,5.4 \%)$. The 10 remaining papers did not report outcomes measures with one $(2.1 \%)$ that reported structure and process indicators only and $9(19.1 \%)$ reported process indicators only.

\section{Resources for care provision: structures}

Structures were poorly reported in all studies, with only two giving some details of multidisciplinary team involvement $[52,61]$, while, none on the pharmacist skill mix or time allocation. The only aspect of structures reported relating to training which was given in five studies. In one, pharmacists and pharmacy residents were engaged in a two-week training of literature review and patient assessments [35]. A community pharmacist based study described a workshop covering clinical presentations of CKD, managing drug-related problems and discussing patient cases [48]. Similar training was described for community pharmacists, [18] and hospital clinical pharmacists [16], to enable them to identify patients with renal insufficiency and perform dose adjustments. A four session course to all members of the multidisciplinary team prior to the study was described in one article [61].

\section{Characteristics of clinical pharmacy practice: processes}

All studies provided some description of the processes undertaken by the pharmacists, although the detail provided varied considerably and was generally lacking. The majority of processes (often labelled as interventions) included medication chart review to identify any drugrelated problems (DRPs) [15-31]. Many studies reported pharmacists' interventions in: modifying drug doses and recommending new pharmacotherapy; $[16,19,21-23$,
25-27, 29, 30, 32-40, 52, 59]; interacting with a member of the multidisciplinary team; [15-17, 19-21, 23-25, 27, $31,32,34-38,40-43]$ requesting and monitoring laboratory parameters; $[15,23,25,27,33,34,36,37,43]$ assessing appropriateness of medications prescribed for hospitalised patients at each point of care; $[17,22,29$, 30, 35-38, 40, 57]. Fewer studies described pharmacist processes at out-patient, pharmacist-led clinics relating to the management of specific CKD complications, such as anaemia; [34, 39, 44] hypertension and diabetes; [54] managing hypertension through telemedicine; [41] optimising dyslipidaemia management; [37, 45] improving haemoglobin A1c levels (HbA1c); [43] and emphasising smoking cessation. [37, 43] Development of protocols and compiling and updating guidelines were also described in two studies [22, 34]. Performing medication reconciliation [46]; providing patient medication counselling, education on disease status or medication, conducting motivational interviews to improve adherence were also reported [15, $25,27,29,30,34,36,37,42,43,47-50,55,57,58,60]$. A number of studies reported pharmacists' participation in ward rounds $[17,22,35,37,38,40]$, providing educational sessions to healthcare professionals $[22,34]$ and performing activities such as medication use evaluations [34]. There were no reports of pharmacist prescribing activities; one study described the process of deprescribing to optimise medication use [59].

Fewer studies provided any data on time spent on specific activities. Interaction time between pharmacist and patients were reported in two studies, varying from 15 to $30 \mathrm{~min}$ [43, $50]$ and the timeframe in which the services were provided ranged from daily [35-38, 40] to every three months [47].

Across all studies, the pharmacists identified 5302 drugrelated problems in 2933 patients. Pharmacists made 3160 recommendations to healthcare professionals with an acceptance rate varying from $33.3 \%$ in a community setting; [16] $46.43 \%$ in a dialysis unit; [59] to around $95 \%$ in hospital settings [17, 24, 42, 51, 52, 57]. Only three studies reported the clinical significance of recommendations. Of these $26 \%$ were of moderate to [29], $48.8 \%$ of major clinical significance [51] and $47 \%$ serious severity [20].

A pharmacist-based quality improvement programme consisting of pharmacists' interactions with the patients and electronic collaboration with the physicians was associated with a significant improvement in the measurement of PTH during the study period [15]. Pharmacists' interventions led to medication therapy modifications [16-21, 24-29, 31, 33, $37,42,46]$ and resolving medication record discrepancies $[46,57]$. Patients' compliance with ongoing blood pressure (BP) monitoring post kidney transplantation was significantly improved with pharmacists' input [41]. Counselling by pharmacists significantly improved medication adherence in patients with CKD [47, 50, 60]. 


\section{Clinical outcomes}

The final column of Tables 1 and 2 titled 'Main outcomes achieved' provides a detailed summary of main results and statistical significance values related to each of the studies summarised below. Clinical outcomes only were reported in $(n=17)$ studies. A pharmacist-based quality improvement programme in a pragmatic randomised controlled study reported that patients in the intervention arm were prescribed more classes of antihypertensive medications than those in the control arm [15]. In a 6-month cluster randomised trial, pharmacists attending a structured training and communication-network programme (ProFil) and managing hypertension in CKD patients demonstrated larger reduction in systolic blood pressure (BP) of the intervention group compared to the usual care group [48].

Intervention in the management of BP in CKD and haemodialysis resulted in achieving target $\mathrm{BP}$ in the intervention versus the control group [32, 54, 55], significant reductions in mean systolic and diastolic BP in a group of kidney transplant recipients [41], and significant reduction in systolic and diastolic BP in diabetic nephropathy [43]. Only one article showed that pharmacists' intervention in an intensive care unit (ICU) setting reduced the length of ICU stay [38]. Another study reported reduction in the length of stay in the intervention group by 1.3 days $(p<0.001)$ and reduced unplanned admission by $27 \%$ ( $p=0.047)$ [52]. One further study showed no difference of pharmacists' intervention compared to usual care on hospital readmission outcomes [57]. Pharmacists were also involved in the monitoring of kidney function in patients with CKD and demonstrated significant differences in measuring $\mathrm{CrCl}$ between discharge and admission [36]. However, one study demonstrated no difference in the mean serum creatinine or estimated glomerular filtration rate (eGFR) between the intervention and control groups [58]. A retrospective controlled study reported improvement in eGFR, uric acid, cholesterol and $\mathrm{HbA1c}$ in the intervention group compared to the control group after multidisciplinary care, however, pharmacists' contribution to the care was not clearly reported [61].

Four studies gave outcomes of pharmacists managing anaemia in CKD patients [34, 39, 44, 55], with significant haemoglobin values within target range in pharmacist-led clinic. Time to achieve target haemoglobin was 28 days in the pharmacist-managed group compared with 41 days in the usual care group [44]. While the proportion of patients achieving target haemoglobin was not significant, pharmacist intervention significantly improved haemoglobin and iron monitoring by improving compliance to therapy [44]. Pharmacist counselling significantly improved haemoglobin levels in one study [34], with haemoglobin concentration and Transferrin saturation (TSAT\%) increasing significantly and serum ferritin reaching target values in a prospective uncontrolled study [23].

An uncontrolled study of the impact of on managing secondary complications of haemodialysis patients resulted in significantly increased median serum calcium in those with hypocalcaemia and decreased values in hypercalcaemia, a decline in serum phosphate in patients with hyperphosphataemia, and an increase and decrease in serum iPTH in patients with sub-optimal and supra-optimal levels respectively [23].

Pharmacists' interventions in a pragmatic, cluster randomised study improved screening of proteinuria between an interventions compared to control group [45]. A nonrandomised controlled study of pharmacist involvement in a monitoring program for CKD reported significant differences in $\mathrm{CrCl}$ between discharge and admission in both the control and intervention groups [36].

\section{Humanistic outcomes}

In a cluster, randomised study health related quality of life (HRQoL) improved significantly compared to control in a group of haemodialysis patients receiving pharmacist intervention over a 6-month period [30]. In a non-randomised controlled study, HRQoL domains were not significantly impacted by the additional pharmacist care in kidney transplants [47]. A multicentre RCT reported significant improvement in HRQoL scores in the intervention group compared to control [53].

Patient satisfaction reported in two randomised controlled studies: $92 \%$ of patients had positive feelings about pharmacists' involvement in their care and felt that the pharmacist provided beneficial information [15] and $43 \%$ of patients were 'very satisfied' with the care received and were willing to receive future care from the pharmacist [45]. A crosssectional prospective study demonstrated that patients were greatly satisfied with the intervention [58].

\section{Economic outcomes}

Only seven studies reported economic outcomes resulting from pharmacist input $[22,35,38-40,44,56]$. One study reported that pharmacists in the ICU could contribute to significant cost savings in septic patients, with antimicrobial prescribing efficiencies accounted for $34.7 \%$ of total savings [38]. In a study investigating an ICU pharmacist dosing adjustment programme, the mean ICU hospitalisation costs per patient decreased significantly with total savings of 2669.5 USD per patient [40]. Jiang et al. demonstrated that pharmacist dosing adjustment resulted in drug cost savings per patient of 2345.98 USD with antibiotics accounting for $64.5 \%$ of all cost savings. The presence of an ICU pharmacist resulted in 2346 USD savings per patient receiving 
continuous renal replacement therapy [35]. Debenito et al. reported that the mean weekly dose of erythropoiesis-stimulating agents (ESAs) was significantly less in the pharmacistmanaged group than the usual care group and the annualised ESA cost per patient reduced by 1288 USD [44], whereas, Aspinall et al. reported lower average dose of darbepoetin in the pharmacist-managed ESA clinic compared to the usual care [39]. Mousavi et al. showed that the cost per patient for inappropriate stress ulcer prophylaxis administration in patients with insufficient renal function was reduced by pharmacists' intervention [22]. A multicentre RCT reported that pharmaceutical care costed more per quality adjusted life year (QALY) gained compared to usual care [56].

\section{Discussion}

There are a number of important key findings that have arisen from this review and these are outlined below. Fortyseven new studies have been published in the intervening 8 year period since a previous similar review [7]. Ten of these are of a 'gold standard' RCT design and the quality of the controlled studies included is generally poor. Structures and processes were very poorly reported and none of the studies included consideration of pharmacist prescribing-which is considered in several countries, where it has been implemented, to be a significant advance in pharmacy practice. The process indicators in the original review [7] and this review were very similar but this review identified papers with clear shift from only identifying drug-related problems to more involvement of the pharmacist in medication therapy management. Most of the studies in this review continue to focus on and report details of DRPs as an indicator of the process of pharmacy practice. Some of these considered the clinical significance of these DRPs but this was not universal. Less focus on clinical, humanistic and economic outcomes was observed in majority of the papers in both reviews.

Many of the uncontrolled studies had a variety of quality deficiencies including; lack of comprehensive explanation of the pharmacists' intervention, under-reporting of adverse events and insufficient information to allow reproduction of the study for interested readers. Few studies lacked some important information leading to poor scoring of the study, such as lack of clarity in stating the study aim, [35] the number of participants, the population from where the sample was drawn, duration of the data collection or the study period, frequency of follow-up, and some studies were unable to clearly state the distribution of the confounders in both groups [15, 22, 30, 35, 39, 45].

The majority of the 20 controlled studies were of 'fair' quality with the exception of four that were considered 'weak' $[22,55,56,58]$. High quality RCTs with low levels of bias generate the highest level of evidence [62]. However, the availability of quality evidence in this area is limited with only 5 RCTs were included in this review and 4 in a previous review by Salgado et al. [7]. The RCTs in both reviews lacked sufficient information on the randomisation process, in addition to poor detail on any blinding process of the care-giver and the care-receiver (however, it might be a challenge to blind in some study designs) so jeopardising the quality of these studies [63]. It is therefore evident that there has been a limited amount of high quality research published for the benefits of clinical pharmacy practice in CKD. There is particularly a paucity of evidence from RCTs offering a robust evidence base for practice. Despite this criticism there is a growing body of information in relation to some aspects of clinical pharmacy practice that offers some insights to the developing quality of services provided making real and significant differences to the outcomes of patients. This, however, needs to be verified through even more robust RCTs that are better resourced, designed and executed.

The gathering of more gold standard evidence such as RCTs is essential to enable measuring the impact of clinical pharmacists' intervention in patients with CKD compared to standard care. Furthermore, there is an identified need to carry out studies with explicit details and accurate definitions including the setting, the participants, the randomisation process and the interventions of interest.

It is of paramount importance that detailed descriptions of the interventions, in terms of structures and processes and outcomes, are included in publications to allow them to be reproduced and for readers to consider the studies within the context of their own practice [64]. Most papers lacked sufficient details of the clinical pharmacy practices so making it difficult to fully understand the activity. Without full insight to practice it is difficult to fully understand the context and characteristics of practice and so reproduce the structures and processes in wider settings. This is not just a deficiency of studies in CKD since a study by Schroter et al. to assess the replicability of published clinical interventions, in a variety of clinical settings, reported that $57 \%$ of the studies had insufficient description of the intervention of interest to make it replicable [65]. A tool produced by Correr et al. to address the lack of intervention descriptions in clinical pharmacy research (Descriptive Elements of Pharmacist Intervention Characterization Tool) DEPICT is a validated instrument for accurately describing the details of pharmacist interventions performed as part of clinical pharmacy practice [66]. This tool could be used as a guidance to structurally describe the intervention of interest in pharmacy practice research.

Additionally it should be noted that in CKD there are no studies that have specifically investigated prescribing as part of clinical pharmacy practice and there are no 
full description of structure, processes and outcomes as they relate to prescribing practice. A systematic review by Tesfaye et al. published in 2017 of the prevalence of inappropriate prescribing and the impact of pharmacists' interventions reported significant reduction in inappropriate prescribing when physicians received immediate concurrent feedback from a clinical pharmacist [67]. The review showed minimal involvement of the pharmacist in the role of prescribing for patients with CKD. Despite the increased recognition of prescribing models such as independent, supplementary or collaborative [6], there was limited published evidence to lead to the best practice model for prescribing.

There is also a need to stimulate more of a research culture within clinical pharmacy practice. A paper by Peterson et al. reported that lack of time, lack of opportunities, lack of training and never being asked to participate in a research were major barriers for pharmacists' engagement in research [68]. A systematic review by Awaisu et al. concluded that pharmacists are aware of the value of research to enable them advance pharmacy practice and indicate their willingness to be involved in independent research and in practice-based research networks. However, lack of time, training and support were the main barriers [69].

A strength for this review is that the protocol was peer reviewed and registered with PROSPERO. The protocol was devised in accordance with PRISMA-P (Preferred Reporting Items for Systematic review and Meta-Analysis Protocols) standards [9] and the systematic review was conducted and reported in accordance with PRISMA (Preferred Reporting Items for Systematic Review and MetaAnalysis) standards [10]. In terms of limitations, publication bias could potentially affect the selecting process of the articles, since no study was identified to show the negative impact of clinical pharmacy services in caring for patients with CKD. One further limitation is the exclusion of papers in languages other than English potentially leading to the omission of relevant papers.

In conducting RCTs, it has been recognised that it is vital to be careful in the selection and recording of outcomes to build up a coherent dataset [70-73]. Moreover, consistency in the use of outcomes will aid future users of the services and those involved in resource allocation, planning and implementation of clinical pharmacy services [72]. It is evident from this review that where RCTs were conducted, there was no consistency in the selection and reporting of outcomes. These issues could be addressed with the development and application of agreed standardised sets of outcomes [73]. Research on core outcome set definitions for clinical pharmacy practice is ongoing in many areas such as polypharmacy [74] but this appears to be lacking in CKD, which could be a potential area of work in the future.

\section{Conclusion}

There is some evidence for the outcomes of pharmacists' intervention in patients with CKD but this is generally of low quality and insufficient volume. The controlled studies in this systematic review showed that pharmacist interventions improved patients' clinical outcomes such as $\mathrm{Hb}$ levels, $\mathrm{CrCl}$, PTH and calcium levels. However, these studies lacked detail on reporting of the humanistic outcomes and there remains a paucity of evidence demonstrating economic impact of pharmacists' interventions.

There is some evidence since the last review that shows positive contributions of pharmacists' involvement in the multidisciplinary team to provide care to patients with CKD. This includes evidence on the structure, processes of care and the outcomes of pharmacists' intervention in patients with CKD. More high-quality research in this area is warranted.

Acknowledgements Ms Tesnime Jebara for input to quality assessment of papers. Mr Hamed Al Naamani for production of graphs and figures and general technical support in production of the manuscript.

Authors' contributions All authors were involved in all aspects of this work including; conception and design, analysis and interpretation of data, drafting and revising the article, providing intellectual content and final approval of the version to be published.

Funding None.

Conflicts of interest None of the authors has any financial interests or connections, direct or indirect, or other situations that might raise the question of bias in the work reported or the conclusions, implications or opinions stated. In addition, the authors confirm that results presented in this paper have not been published previously in whole or part, except in abstract format.

Ethics approval The Ethics panel of the School of Pharmacy \& Life Sciences, Robert Gordon University indicated that ethics approval was not required for this systematic review.

Open Access This article is distributed under the terms of the Creative Commons Attribution 4.0 International License (http://creativeco mmons.org/licenses/by/4.0/), which permits unrestricted use, distribution, and reproduction in any medium, provided you give appropriate credit to the original author(s) and the source, provide a link to the Creative Commons license, and indicate if changes were made.

\section{References}

1. KDIGO clinical practice guideline for the evaluation and Management of chronic kidney disease. [online] Belgium: the International Society of Nephrology. http://www.kdigo.org/clini cal_practice_guidelines/pdf/CKD/KDIGO_2012_CKD_GL.pdf (2013). Accessed 07 Aug 2017. 
2. Crockell YJ. Management of chronic kidney disease: an emphasis on delaying disease progression and treatment options. Formulary. 2012;47(6):228-30.

3. McBane SE, Dopp AL, Abe A, Benavides S, Chester EA, Dixon DL, et al. Collaborative drug therapy management and comprehensive medication management. Pharmacotherapy. 2015, 35(4), e39-50. https://doi.org/10.1002/phar.1563.

4. Mason NA, Bakus JL. Strategies for reducing polypharmacy and other medication-related problems in Chronic Kidney Disease. Semin Dial. 2010; 55-1.

5. Chisholm MA, Mulloy LL, Jagadeesan M, DiPiro JT. Impact of clinical pharmacy services on renal transplant patients' compliance with immunosuppressive medications. Clin Transplant. 2001;15(5):330-6.

6. Royal pharmaceutical society. A competency framework for all prescribers. https://www.rpharms.com/Portals/0/RPS\%20doc ument $\% 20$ library/Open\%20access/Professional\%20standards/ Prescribing $\% 20$ competency $\% 20$ framework/prescribing-compe tency-framework.pdf (2016). Accessed 23 Jan 2017.

7. Salgado TM, Moles R, Benrimoj SI, Fernandez-Llimos F. Pharmacists' interventions in the management of patients with chronic kidney disease: a systematic review. Nephro Dial Transplant. 2012;27(1):276-92.

8. Kozma CM, Reeder CE, Schulz RM. Economic, clinical, and humanistic outcomes: a planning model for pharmacoeconomic research. Clin Ther. 1993;15:1121-32.

9. Moher D, Shamseer L, Clarke M, Ghersi D, Liberati A, Petticrew $\mathrm{M}$, et al. Preferred reporting items for systematic review and meta-analysis protocols (PRISMA-P) 2015 statement. Syst Rev. 2015;4(1):1. https://doi.org/10.1186/2046-4053-4-1.

10. Moher D, Liberati A, Tetzlaff J, Altman DG. Preferred reporting items for systematic reviews and meta-analyses: the PRISMA statement. Int J Surg. 2010;8(5):336-41.

11. Pluye P, Robert E, Cargo M, Bartlett G, O'Cathain A, Griffiths F, et al. Proposal: a mixed methods appraisal tool for systematic mixed studies reviews. [online]. Montreal: McGill University http://mixedmethodsappraisaltoolpublic.pbworks.com/w/file/ fetch/84371689/MMAT\%202011\%20criteria\%20and\%20tutorial \%202011-06-29updated2014.08.21.pdf (2011). Accessed 03 Aug 2017.

12. Downs SH, Black N. The feasibility of creating a checklist for the assessment of the methodological quality both of randomized and non-randomized studies of health care interventions. J Epidemiol Commun Health. 1998;52:377-84.

13. Machado M, Bajcar J, Guzzo GC, Einarson TR. Sensitivity of patient outcomes to pharmacist interventions. Part I: systematic review and meta-analysis in diabetes management. Ann Pharmacother. 2007;41:1569-82.

14. Donabedian A. The quality of care: How can it be assessed? JAMA. 1988;260(12):1743-8.

15. Cooney D, Moon H, Liu Y, Miller RT, Perzynski A, Watts B, et al. A pharmacist based intervention to improve the care of patients with CKD: a pragmatic, randomized, controlled trial. Bio Med Central Nephrol. 2015;16(1):56.

16. Pourrat X, Sipert AS, Gatault P, Sautenet B, Hay N, Guinard F, et al. Community pharmacist intervention in patients with renal impairment. Int J Clin Pharm. 2015;37(6):1172-9.

17. Vessal G. Detection of prescription errors by a unit-based clinical pharmacist in a nephrology ward. Pharm World Sci. 2010;32(1):59-65.

18. Via-Sosa MA, Lopes N, March M. Effectiveness of a drug dosing service provided by community pharmacists in polymedicated elderly patients with renal impairment-a comparative study. BMC Fam Pract. 2013;14:96.

19. Gheewala PA, Peterson GM, Curtain CM, Nishtala PS, Hannan PJ, Castelino RL. Impact of the pharmacist medication review services on drug-related problems and potentially inappropriate prescribing of renally cleared medications in residents of aged care facilities. Drugs Aging. 2014;31(11):825-35.

20. Staino C, Pilch N, Patel S, Trobaugh K, Fleming J, Meadows $\mathrm{H}$, et al. Optimizing finite resources: pharmacist chart reviews in an outpatient kidney transplant clinic. J Am Pharm Assoc. 2015;55(6):613-20.

21. Belaiche S, Romanet T, Allenet B, Calop J, Zaoui P. Identification of drug-related problems in ambulatory chronic kidney disease patients: a 6-month prospective study. J Nephrol. 2012;25(5):782-8.

22. Mousavi M, Dashti-Khavidaki S, Khalili H, Farshchi A, Gatmiri M. Impact of clinical pharmacy services on stress ulcer prophylaxis prescribing and related cost in patients with renal insufficiency. Int J pharm pract. 2013;21(4):263-9.

23. Dashti-Khavidaki S, Khalili H, Shahverdi S, Abbasi MR, Lessan-Pezeshki $\mathrm{M}$. The role of clinical pharmacy services in achieving treatment targets in Iranian haemodialysis patients. Singapore Med J. 2012;53(9):599-603.

24. Holm H, Bjerke K, Holst L, Mathiesen L. Use of renal risk drugs in patients with renal impairment. Int $\mathrm{J}$ clin pharm. 2015;37(6):1136-42.

25. Chen LL. A preliminary review of the medication management service conducted by pharmacists in haemodialysis patients of Singapore General Hospital. Proceedings of Singapore Healthcare. 2013;22(2):103-6.

26. Arrabal-Durán P, Durán-García ME, Ribed-Sánchez A, Hidalgo-Collazos P, Sanjurjo-Sáez M. Pharmaceutical interventions in prescriptions for patients admitted with chronic renal failure. Nefrologia. 2014;34(6):710-5. https://doi.org/10.3265/ Nefrologia.pre2014.Jul.12541.

27. Barnes KD, Tayal NH, Lehman AM, Beatty SJ. Pharmacistdriven renal medication dosing intervention in a primary care patient-centered medical home. Pharmacotherapy. 2014;34(12):1330-5.

28. Belaiche S, Romanet T, Bell R, Calop J, Allenet B, Zaoui P. Pharmaceutical care in chronic kidney disease: experience at Grenoble University Hospital from 2006 to 2010. J Nephrol. 2012;25(4):558.

29. Castelino RL, Sathvik BS, Parthasarathi G, Gurudev KC, Shetty MS, Narahari MG. Prevalence of medication-related problems among patients with renal compromise in an Indian hospital. J Clin Pharm Ther. 2011;36(4):481-7.

30. Dashti-Khavidaki S, Sharif Z, Khalili H, Badri S, Alimadadi A, Ahmadi F, et al. The use of pharmaceutical care to improve health-related quality of life in hemodialysis patients in Iran. Int J Clin Pharm. 2013;35(2):260-7.

31. Ramadaniati HU, Anggriani Y, Wowor V, Rianti A. Drug-related problems in chronic kidneys disease patients in an Indonesian hospital: Do the problems really matter? Int J Pharm Phar Sci. 2016;8(12):298-302.

32. Qudah B, Albsoul-Younes A, Alawa E, Mehyar N. Role of clinical pharmacist in the management of blood pressure in dialysis patients. Int J Clin Pharm. 2016;38(4):931-40.

33. Geerts AF, Haan ND, Koning FH, Sterren TM, Weel CV, Vervoort GM, et al. A pharmacy medication alert system based on renal function in older patients. Br J Gen Pract. 2012;62(601):e525-9.

34. Ohnishi J, Miyake A, Kuwatsuka K, Onoue Y, Lee M, Koyama $\mathrm{T}$, et al. Effect of pharmacist management on serum hemoglobin levels with renal anemia in hemodialysis outpatients. Biol Pharm Bull. 2011;34(10):1609-12.

35. Jiang SP, Zhu ZY, Wu XL, Lu XY, Zhang XG, Wu BH. Effectiveness of pharmacist dosing adjustment for critically ill patients receiving continuous renal replacement therapy: a comparative study. Ther Clin Risk Manag. 2014;10(1):405-12. 
36. Cabello-Muriel A, Gascón-Cánovas JJ, Urbieta-Sanz E, Iniesta-Navalón C. Effectiveness of pharmacist intervention in patients with chronic kidney disease. Int J Clin Pharm. 2014;36(5):896-903.

37. AbuRuz SM, Alrashdan Y, Jarab A, Jaber D, Alawwa IA. Evaluation of the impact of pharmaceutical care service on hospitalized patients with chronic kidney disease in Jordan. Int J Clin Pharm. 2013;35(5):780-9.

38. Jiang SP, Zhu ZY, Ma KF, Zheng X, Lu XY. Impact of pharmacist antimicrobial dosing adjustments in septic patients on continuous renal replacement therapy in an intensive care unit. Scand J Infect Dis. 2013;45(12):891-9.

39. Aspinall SL, Cunningham FE, Zhao X, Boresi JS, Tonnu-Mihara IQ, Smith KJ, et al. Impact of pharmacist-managed erythropoiesisstimulating agents clinics for patients with non-dialysis-dependent CKD. Am J Kidney Dis. 2012;60(3):371-9.

40. Jiang SP, Xu YY, Yang P, Wu WF, Zhang XG, Lu XY, et al. Improving antimicrobial dosing in critically ill patients receiving continuous venovenous hemofiltration and the effect of pharmacist dosing adjustment. Eur J Intern Med. 2014;25(10):930-5.

41. Aberger EW, Migliozzi D, Follick MJ, Malick T, Ahern DK. Enhancing patient engagement and blood pressure management for renal transplant recipients via home electronic monitoring and webenabled collaborative care. Telemed J E Health. 2014;20(9):850-4.

42. Adibe MO, Igboeli NU, Ukwe CV. Evaluation of drug therapy problems among renal patients receiving care in some tertiary hospitals in Nigeria. Trop J Pharm Res. 2017;16(3):697-704.

43. Kelly CJ, Booth G. Pharmacist-led structured care for patients with diabetic nephropathy. Br J Diabetes Vasc Dis. 2008;8(2):86-8.

44. Debenito JM, Billups SJ, Tran TS, Price LC. Impact of a clinical pharmacy anemia management service on adherence to monitoring guidelines, clinical outcomes, and medication utilization. J Manag Care Spec Pharm. 2014;20(7):715-20.

45. Chang AR, Evans M, Yule C, Bohn L, Young A, Lewis M, et al. Using pharmacists to improve risk stratification and management of stage 3A chronic kidney disease: a feasibility study. BMC Nephrol. 2016;17(1):168.

46. Patricia NJ, Foote EF. A pharmacy-based medication reconciliation and review program in hemodialysis patients: a prospective study. Pharm Pract. 2016;14(3):785. https://doi.org/10.18549/Pharmpract .2016 .03 .785

47. Joost R, Dörje F, Schwitulla J, Eckardt KU, Hugo C. Intensified pharmaceutical care is improving immunosuppressive medication adherence in kidney transplant recipients during the first post-transplant year: a quasi-experimental study. Nephrol Dial Transplant. 2014;29(8):1597-607.

48. Santschi V, Lord A, Berbiche D, Lamarre D, Corneille L, Prud'homme L, et al. Impact of collaborative and multidisciplinary care on management of hypertension in chronic kidney disease outpatients. J Pharm Health Serv Res. 2011;2(2):79-87.

49. Venkateswararao S, Asha Sara S, Kshama I, Rama P. Evaluation and pharmacists intervention for improving adherence among renal failure patients. Int J Pharm Pharm Sci. 2015;7(3):82-5.

50. Rani V, Soundararajan P, Samyuktha CH, Kannan G, Thennarasu $\mathrm{P}$. Impact of clinical pharmacist provided education on medication knowledge and adherence of hemodialysis patients in a South Indian university hospital. Asian J Pharm Clin Res. 2013;6:24-7.

51. Dashti-Khavidaki S, Khalili H, Hamishekar H, Shahverdi S. Clinical pharmacy services in an Iranian teaching hospital: a descriptive study. Pharm World Sci. 2009;31(6):696-700.

52. Chia B, Cheen M, Gwee X, Chia BY, Cheen MH, Gwee XY, et al. Outcomes of pharmacist-provided medication review in collaborative care for adult Singaporeans receiving hemodialysis. Int J Clin Pharm. 2017;39:1031-8.

53. Mateti U, Nagappa A, Attur R, Nagaraju SP, Rangaswamy D. Impact of pharmaceutical care on the health-related quality of life among hemodialysis patients-a multicenter randomized controlled study. Saudi J Kidney Dis Transpl. 2017;28(6):1293-306.

54. Anderegg M, Gums T, Uribe L, MacLaughlin EJ, Hoehns J, Bazaldua OV, et al. Pharmacist intervention for blood pressure control in patients with diabetes and/or Chronic Kidney Disease. Pharmacotherapy. 2018;38:309-18.

55. Mateti U, Nagappa A, Attur R, Nagaraju SP, Rangaswamy D. Impact of pharmaceutical care on clinical outcomes among hemodialysis patients: a multicenter randomized controlled study. Saudi J Kidney Dis Transpl. 2018;29:801.

56. Mateti U, Nagappa A, Attur R, Nagaraju SP, Rangaswamy D. Costeffectiveness of pharmaceutical care on patients undergoing maintenance hemodialysis - a multicenter randomized controlled study. Postgrad Med. 2018;130(7):621-6.

57. Tuttle K, Alicic R, Short R, Neumiller JJ, Gates BJ, Daratha KB, et al. Medication therapy management after hospitalization in CKD: a randomized clinical trial. Clin J Am Soc Nephrol. 2018;13:23141.https://doi.org/10.2215/cjn.06790617.

58. Xu X, Feng Y, Tian Y, Wang H. Pharmaceutical care in kidney transplant recipients: behavioral and physiologic outcomes at 12 months. Transpl Proc. 2018;50(8):2451-6.

59. Alshamrani M, Almalki A, Qureshi M, Yusuf O, Ismail S. Polypharmacy and medication-related problems in hemodialysis patients: a call for deprescribing. Pharmacy. 2018;6:76-85.

60. Chandrasekhar D, Ganesan V, Sreekumar S, Pradeep A, Geoji AS, George AE, et al. Impact of intensified pharmaceutical interventions in medication adherence in chronic kidney disease patients. J Young Pharm. 2018;10(2):208-12.

61. Imamura $Y$, Takahashi $Y$, Hayashi T, Iwamoto M, Nakamura R, Goto M, et al. Usefulness of multidisciplinary care to prevent worsening renal function in chronic kidney disease. Clin Exp Nephrol. 2018;19:1-9.

62. Burns PB, Rohrich RJ, Chung KC. The levels of evidence and their role in evidence-based medicine. Plast Reconstr Surg. 2011;128(1):305-10.

63. Mahboobi H, Khorgoei T, Bansal N. Designing, conducting and reporting randomised controlled trials: a few key points. ISBN: 978953-51-0504-6, In Tech 2012. http://www.intechopen.com/books /evidence-based-medicine-closer-to-patients-or-scientists-/desig ningconducting-and-reporting-rtcs-key-points. Accessed $12 \mathrm{Feb}$ 2018.

64. Salgado TM, Correr CJ, Moles R, Benrimoj SI, Fernandez-Llimos F. Assessing the implementability of clinical pharmacist interventions in patients with chronic kidney disease: an analysis of systematic reviews. Ann Pharmacother. 2013;47(11):1498-506.

65. Schroter S, Glasziou P, Heneghan C. Quality of descriptions of treatments: a review of published randomised controlled trials. BMJ Open. 2012;2:e01978.

66. Correr CJ, Melchiors AC, Souza TT, Rotta I, Salgado TM, Fernandez-Llimos F. A tool to characterize the components of pharmacist interventions in clinical pharmacy services: the DEPICT Project. Ann Pharmacother. 2013;47:946-52. https://doi.org/10.1345/ aph.1S006.

67. Tesfaye WH, Castelino RL, Wimmer BC, Zaidi STR. Inappropriate prescribing in chronic kidney disease: a systematic review of prevalence, associated clinical outcomes and impact of interventions. Int J Clin Pract. 2017. https://doi.org/10.1111/ijcp.12960.

68. Peterson GM, Jackson SL, Fitzmaurice KD, Gee PR. Attitudes of Australian pharmacists towards practice-based research. J Clin Pharm Ther. 2009;34(4):397-405. https://doi.org/10.111 1/j.1365-2710.2008.01020.x.

69. Awaisu A, Alsalimy N. Pharmacists' involvement in and attitudes towards pharmacy practice research: a systematic review of the literature. Res Social Adm Pharm. 2014. https://doi.org/10.1016/j. sapharm.2014.12.008. 
70. Esposito P, Dal Canton A. Clinical audit, a valuable tool to improve quality of care: general methodology and applications in nephrology. World J Nephrol. 2014;3(4):249-55. https://doi.org/10.5527/ wjn.v3.i4.249.

71. Beuscart JB, Knol W, Cullinan S, Schneider C, Dalleur O, Boland $\mathrm{B}$, et al. International core outcome set for clinical trials of medication review in multi-morbid older patients with polypharmacy. BMC Med. 2018;16(1):21-9.

72. Lombardi N, Wei L, Ghaleb M, Pasut E, Leschiutta S, Rossi P, et al. Evaluation of the implementation of a clinical pharmacy service on an acute internal medicine ward in Italy. BMC Health Serv Res. 2018;18:259. https://doi.org/10.1186/s12913-018-2988-y.

73. Williamson PR, Altman DG, Bagley H, Barnes KL, Blazeby JM, Brookes ST, et al. The COMET handbook: version 1.0. Trials 2017;18(Suppl 3):280.
74. Rankin A, Cadogan CA, Ryan C, Clyne B, Smith SM, Hughes CM, et al. Core outcome set for trials aimed at improving appropriate polypharmacy in older people in primary care. J Am Geriatr Soc. 2018. https://doi.org/10.1111/jgs.15245.

Publisher's Note Springer Nature remains neutral with regard to jurisdictional claims in published maps and institutional affiliations. 